\title{
Nonlinear orthogonal projection
}

\author{
by Ewa Dudek and Konstanty Holly (Kraków)
}

\begin{abstract}
We discuss some properties of an orthogonal projection onto a subset of a Euclidean space. The special stress is laid on projection's regularity and characterization of the interior of its domain.
\end{abstract}

0. Introduction. Let $M$ be a non-empty subset of a metric space $Z$. We define a relation $\mathcal{P} \subset Z \times M$, which we call the orthogonal projection onto $M$. Its domain is

$\operatorname{dom} \mathcal{P}:=\left\{z \in Z:\right.$ there exists a unique point $z^{\prime} \in M$

$$
\text { such that } \left.d\left(z, z^{\prime}\right)=\varrho(z, M)\right\},
$$

where $d$ denotes the metric of $Z$ and $\varrho(z, M):=\inf _{x \in M} d(z, x)$. Obviously, $M \subset \operatorname{dom} \mathcal{P}$. The orthogonal projection of $z \in \operatorname{dom} \mathcal{P}$ is defined to be the unique point $\left(z^{\prime}=\right) \mathcal{P}(z) \in M$ which realizes the distance of $z$ to $M$. If $M$ is a closed linear subspace of a Hilbert space $Z$, then $\mathcal{P}$ is the well-known linear orthogonal projection: $Z=\operatorname{dom} \mathcal{P} \rightarrow M$.

The need of considering orthogonal projections onto non-linear sets has been noticed since a long time. For example, if $Z=\mathbb{R}^{n}$ and $M$ is a smooth (or analytic) submanifold, then the composition $\left.f \circ \mathcal{P}\right|_{\text {int dom } \mathcal{P}}$ is the most natural smooth (analytic) extension of a given smooth (analytic) function $f: M \rightarrow \mathbb{R}$ on an open neighbourhood of $M$ (because in this case $M \subset$ int $\operatorname{dom} \mathcal{P}$ (see the generalization (3.8) of the classical result of Federer [5] and (4.1))). Of course, there are other methods of extending such functions, e.g. in the non-analytic case by local straightening of $M$ or by applying Whitney's theory. However, in numerous problems the extension $f \circ \mathcal{P}$ is most useful, since it is simple and effective. The set int $\operatorname{dom} \mathcal{P}$ is in some sense a star-shaped neighbourhood of $M$ (see (1.5), (3.13)), so the retraction $\left.\mathcal{P}\right|_{\text {int dom } \mathcal{P}}$ is helpful in studies on differentiable homotopy, e.g. for a given solenoidal vector field $v: G \rightarrow \mathbb{R}^{n}$ vanishing on the boundary of a

1991 Mathematics Subject Classification: 58B10, 51Kxx.

Key words and phrases: projection's regularity and interior of its domain. 
domain $G \subset \mathbb{R}^{n}$ one can construct — with the aid of $\mathcal{P}$ —a sequence $\left(\Phi_{\nu}\right)_{\nu=1}^{\infty}$ of solenoidal vector fields on $G$ equal to zero in a neighbourhood of $M=\partial G$ such that $\Phi_{\nu} \rightarrow v$ together with derivatives as $\nu \rightarrow \infty$.

The notion of the nonlinear orthogonal projection enables us to formulate a new, curvilinear version of the theorem on the existence of the Fréchet differential (see $\left.\left(4^{\prime} .13\right)\right)$.

Furthermore, the differential properties of the distance function $z \mapsto$ $|z-\mathcal{P}(z)|=\varrho(z, M)$ are useful (see e.g. the lemma of Hopf in Lions [13] (Part 1, Lemma 7.2) or Hopf [8] or applications of $\varrho$ in Serrin [16] and Gilbarg-Trudinger [6]).

Literature we know contains only studies on restrictions of orthogonal projections onto submanifolds (of a Riemannian manifold $Z$ ) to small neighbourhoods of $M$ (e.g. the tubular neighbourhood theorem in Hirsch [7]). In this paper we present various properties of the mapping $\mathcal{P}$ without assuming that $M$ is a submanifold. These are topological properties; for example, we formulate a criterion for $z(\in \operatorname{dom} \mathcal{P})$ to be an interior point of $\operatorname{dom} \mathcal{P}$ (see $(2.8)$ ). In so general a situation one cannot expect the orthogonal projection to be differentiable; G. Jasiński [9] proved that the class $M$ of values of a $C^{1}$-retraction of a domain $U \in$ top $\mathbb{R}^{n}$ is a differentiable submanifold (of $\mathbb{R}^{n}$ ) whenever $M \in \operatorname{cotop} U$ (i.e. $M$ is closed in $U$ ). In the present work we wish to investigate the orthogonal projection globally; in particular, almost all theorems we formulate refer also to arguments $z(\in \operatorname{dom} \mathcal{P})$ which may lie at a large distance from $M$.

The theorems presented here can, for the most part, be modified for the case of a Riemannian manifold $Z$. Nevertheless, we restrict our attention to the basic situation $Z=\mathbb{R}^{n}$.

1. Projection onto an arbitrary subset of a Euclidean space. Let $Z$ denote a Euclidean space, i.e. a real finite-dimensional Hilbert space (e.g. $Z=\mathbb{R}^{n}$ ) with a scalar product $(\cdot \mid \cdot)$, which defines the norm $|\cdot|$. From now on $\Omega$ stands for the interior of the domain of the projection $\mathcal{P}$.

(1.1) Example. If $M$ is the unit sphere of $Z$, then

$$
\operatorname{dom} \mathcal{P}=Z \backslash\{0\} \quad \text { and } \quad \forall z \in \operatorname{dom} \mathcal{P}: \mathcal{P}(z)=z /|z| .
$$

Generally, if $M$ is the unit sphere of a linear subspace $Y$, then

$$
\Omega=\operatorname{dom} \mathcal{P}=Z \backslash \operatorname{ker} P_{Y}=Z \backslash Y^{\perp} \text { and } \mathcal{P}(z)=P_{Y}(z) /\left|P_{Y}(z)\right|
$$

for any $z \in \operatorname{dom} \mathcal{P}$, where $P_{Y}$ is the usual projection $Z \rightarrow Y$.

In general, neither has $\operatorname{dom} \mathcal{P}$ to be open, nor $\mathcal{P}$ to be a continuous mapping, even if $M$ is an analytic submanifold: 
(1.2) Example. If $Z=\mathbb{R}^{2}$ and $M=\{(x, y):|y|=1\} \backslash\{(0,1)\}$, then $(0,0) \in(\operatorname{dom} \mathcal{P}) \backslash \Omega$ and $\mathcal{P}$ is not continuous at $(0,0)$.

(1.3) Theorem. The restriction $\left.\mathcal{P}\right|_{\Omega}$ is continuous.

Theorems (lemmas, examples etc.) from Section $k$ are proved or discussed in Section $k^{\prime}, k=1, \ldots, 6$.

(1.4) Corollary. $M \cap \Omega$ is closed in $\Omega$.

(1.5) Theorem. If $a \in Z, a^{\prime} \in M$ and $\left|a-a^{\prime}\right|=\varrho(a, M)$, then:

(i) $\left.] a, a^{\prime}\right] \subset \operatorname{dom} \mathcal{P}$ and $\left.\left.\forall z \in\right] a, a^{\prime}\right]: \mathcal{P}(z)=a^{\prime}$,

(ii) $[a, \mathcal{P}(a)[\subset \Omega$ for $a \in \Omega$.

In general, $\mathcal{P}(a) \notin \Omega$, for example when $Z=\mathbb{R}^{2}, M=\{(x, y): y=|x|\}$ and $a=(0,-1)$.

(1.6) Corollary. Suppose that $f: \mathcal{O} \rightarrow M$ is a continuous mapping of an open set $\mathcal{O} \subset Z$. Furthermore, assume that $\forall z \in \mathcal{O}:|z-f(z)|=\varrho(z, M)$. Then $f \subset \mathcal{P}$; in particular, $\mathcal{O} \subset \Omega$.

Finally, let us mention that the projection is invariant with respect to isometries:

(1.7) Remark. Let $I: Z \rightarrow Z$ be an isometry. Then $I \circ \mathcal{P} \circ I^{-1}$ is the orthogonal projection onto $I(M)$.

2. Closedness of a set near a point. We say that a set $M$ is closed near $z \in Z$ iff

$$
\exists r>\varrho(z, M): \quad M \cap \bar{B}(z, r) \in \operatorname{cotop} Z,
$$

where $B(z, r):=\{x \in Z:|x-z|<r\}$ and $\bar{B}(z, r)=\overline{B(z, r)}$ (Fig. 1).

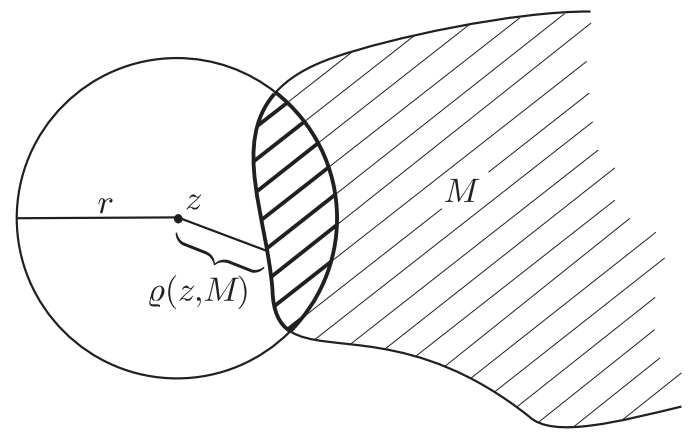

Fig. 1 
(2.2) FACT. If $M$ is closed near $z$, then there exists at least one element of $M$ which realizes the distance $\varrho(z, M)$.

(2.3) FACT. The set $D:=\{z \in Z: M$ is closed near $z\}$ is open in $Z$.

It is obvious that $D=Z$ whenever $M \in \operatorname{cotop} Z$. Then $\operatorname{dom} \mathcal{P}$ is dense in $Z$. This results from the following general

(2.4) Remark. $D \subset \overline{\operatorname{dom} \mathcal{P}}$.

And here is a kind of completing of Theorem (1.3):

(2.5) Remark. The restriction $\left.\mathcal{P}\right|_{D \cap \operatorname{dom} \mathcal{P}}$ is continuous. In particular, $\mathcal{P}$ is continuous whenever $M$ is closed.

(2.6) Proposition. If $M$ is locally compact, then $\Omega \subset D$.

In general, $D \cap \operatorname{dom} \mathcal{P} \not \subset \Omega$, even if $M$ is an analytic submanifold. For $Z=\mathbb{R}^{2}$ and $M=\left\{(x, y): y=x^{2}\right\}$ the point $(0,1 / 2)$ is in $(D \cap \operatorname{dom} \mathcal{P}) \backslash \Omega$ (see $(6.1))$.

Now we formulate a counterpart of Theorem (1.5):

(2.7) Theorem. Suppose that $M$ is locally compact, $a \in Z, a^{\prime} \in M$ and $\left|a-a^{\prime}\right|=\varrho(a, M)$. Then $\left.] a, a^{\prime}\right] \subset D$.

The next theorem is a criterion for being an interior point of the projection's domain.

(2.8) Theorem. Let $M$ be closed near $a \in \operatorname{dom} \mathcal{P}$. Fix $t \in] 0,1]$. Then the following conditions are equivalent:

(i) $a \in \Omega$;

(ii) $\mathcal{P}(a)+t(a-\mathcal{P}(a)) \in \Omega$ and the mapping

$$
w \mapsto w-(1-t) \mathcal{P}(w)
$$

is an injection on a neighbourhood of $\mathcal{P}(a)+t(a-\mathcal{P}(a))$.

3. Projection onto a submanifold. We start with recalling a wellknown property of the tangent space to a submanifold:

(3.1) Remark. If $M$ is a $C^{1}$-submanifold of $Z$ and $a \in Z$ and $a^{\prime} \in M$ satisfy $\left|a-a^{\prime}\right|=\varrho(a, M)$, then

$$
a-a^{\prime} \perp T_{a^{\prime}} M,
$$

where $T_{a^{\prime}} M$ denotes the tangent space to $M$ at $a^{\prime}$. In particular, $\forall a \in$ $\operatorname{dom} \mathcal{P}: a \in \mathcal{P}(a)+\left(T_{\mathcal{P}(a)} M\right)^{\perp}$.

For $z \in M$ and $r>0$ we define the disc of radius $r$ with center at $z$ orthogonal to $M$ by

$$
K_{z}(r):=z+\left\{\zeta \in\left(T_{z} M\right)^{\perp}:|\zeta|<r\right\} .
$$


(3.2) Theorem. Let $M$ be a $C^{1}$-submanifold such that

the mapping: $M \ni z \mapsto T_{z} M \in \mathcal{E}$ satisfies locally the Lipschitz condition in the Hausdorff metric (in the set $\mathcal{E}$ of all non-zero linear subspaces of $Z$ ).

Then for any compact subset $F \subset M$ there is $r>0$ such that:

(i) $\forall y, z \in F:\left\{y \neq z \Rightarrow K_{y}(r) \cap K_{z}(r)=\emptyset\right\}$;

(ii) $\forall z \in F: K_{z}(r) \subset \operatorname{dom} \mathcal{P},\left.\mathcal{P}\right|_{K_{z}(r)} \equiv z$.

The Hausdorff distance of two non-zero linear subspaces $A, B$ is, by definition, the number

$$
d(A, B):=d(A \cap S, B \cap S),
$$

where $S:=\{x \in Z:|x|=1\}$ and $d(A \cap S, B \cap S)$ is the usual Hausdorff distance of the compact sets $A \cap S, B \cap S$. Note that the metric (3.4) defines the usual topology on the Grassmann manifold $\mathcal{G}_{k}(Z)$ of all $k$-dimensional linear subspaces of $Z$; moreover,

$$
\begin{aligned}
& \mathcal{G}_{k}(Z) \ni A \mapsto A^{\perp} \in \mathcal{G}_{N-k}(Z)(1 \leq k \leq N-1, N=\operatorname{dim} Z) \text { is an } \\
& \text { isometry in this metric. }
\end{aligned}
$$

One can also prove that

$$
\text { all } C^{2} \text {-submanifolds satisfy (3.3). }
$$

Submanifolds of class $C^{1}$ generally do not:

(3.7) Example. The curve $M:=\left\{\left(t, \frac{2}{3}|t|^{3 / 2}\right): t \in \mathbb{R}\right\}$ is a $C^{1}$-submanifold of $Z=\mathbb{R}^{2}$. One can check that

$$
\forall r>0 \exists z \in M \backslash\{0\}: \quad K_{z}(r) \cap K_{0}(r) \neq \emptyset .
$$

In particular, (3.3) is not satisfied.

The following theorem is important in the local analysis of a nonlinear orthogonal projection:

(3.8) TheOREM. Let $M$ be a $C^{1}$-submanifold satisfying (3.3). Then for $a \in M$ there exists an open $\mathcal{O} \subset Z$ such that $a \in \mathcal{O} \subset \operatorname{dom} \mathcal{P}$ and

$$
\forall z \in \mathcal{O} \forall x \in \mathcal{O} \cap M: \quad\left\{z-x \perp T_{x} M \Rightarrow \mathcal{P}(z)=x\right\} .
$$

In particular, $M \subset \Omega$.

This is a generalization of Federer's theorem from [5] which states that $M \subset \Omega$ whenever $M$ is a hypersurface of class $C^{2}$.

(3.9) Corollary. If $M$ is a $C^{1}$-submanifold satisfying (3.3), then $\forall a \in \Omega \exists H \in \operatorname{top}\left(T_{\mathcal{P}(a)} M\right)^{\perp}: \quad 0 \in H$ and $\left.\mathcal{P}\right|_{a+H} \equiv \mathcal{P}(a)$. 
(3.10) Corollary. Suppose that $M$ is a $C^{1}$-submanifold satisfying (3.3) and closed near $a \in \operatorname{dom} \mathcal{P}$. Let $\phi: \mathcal{O} \rightarrow M$ be a continuous map on a neighbourhood $\mathcal{O}$ of a such that

$$
\phi(a)=\mathcal{P}(a) \quad \text { and } \quad \forall z \in \mathcal{O}: \quad z-\phi(z) \perp T_{\phi(z)} M .
$$

Then $a \in \Omega$ and $\phi=\mathcal{P}$ in a neighbourhood of $a$.

This result is a differential counterpart of Corollary (1.6). And here is a differential counterpart of the criterion (2.8):

(3.11) Theorem. Let $M$ be a $C^{2}$-submanifold. Fix $z_{0} \in \operatorname{dom} \mathcal{P}$ and an inverse chart $f \subset \mathbb{R}^{n} \times M$ of $M$ which takes on the value $\mathcal{P}\left(z_{0}\right)$. Consider the matrix

$$
A_{f}\left(z_{0}\right):=\left[\left(\frac{\partial f}{\partial x_{i}}\left(x_{0}\right) \mid \frac{\partial f}{\partial x_{j}}\left(x_{0}\right)\right)+\left(\mathcal{P}\left(z_{0}\right)-z_{0} \mid \frac{\partial^{2} f}{\partial x_{i} \partial x_{j}}\left(x_{0}\right)\right)\right],
$$

where $x_{0}=f^{-1}\left(\mathcal{P}\left(z_{0}\right)\right)$. Then the following conditions are equivalent:

(i) $z_{0} \in \Omega$;

(ii) $M$ is closed near $z_{0}$ and $\operatorname{det} A_{f}\left(z_{0}\right) \neq 0$.

The additional assumption " $M \in C^{2}$ " lets us strengthen the conclusion of Theorem (1.5):

(3.13) Theorem. Let $M$ be a $C^{2}$-submanifold. Then

(a) $\left.] a, a^{\prime}\right] \subset \Omega$ for all $\left(a, a^{\prime}\right) \in Z \times M$ such that $\left|a-a^{\prime}\right|=\varrho(a, M)$;

(b) for every $\left(a, a^{\prime}\right) \in(\operatorname{dom} \mathcal{P}) \times M$ the following conditions are equivalent:

(i) $\mathcal{P}(a)=a^{\prime}$;

(ii) $\left.] a, a^{\prime}\right] \subset \Omega$ and $a-a^{\prime} \perp T_{a^{\prime}} M$.

(3.14) Corollary. If $M$ is a $C^{2}$-submanifold, then $\Omega$ is dense in $\operatorname{dom} \mathcal{P}$. If $M$ is also closed in $Z$, then $\bar{\Omega}=Z$ (compare with (2.4)).

\section{Differentiability of an orthogonal projection}

(4.1) Theorem. Let $M$ be a $C^{k}$-submanifold of a Euclidean space $Z$, where $k \in\{2,3, \ldots, \infty, \omega\}$ and $C^{\omega}$ denotes $\mathbb{R}$-analyticity. Then $\left.\mathcal{P}\right|_{\Omega}$ is of class $C^{k-1}$ (as usual $\infty-1:=\infty, \omega-1:=\omega$ ) and

(i) for all $z \in M$, the Fréchet differential $d_{z} \mathcal{P}$ is the linear orthogonal projection onto $T_{z} M$;

(ii) $\forall z \in \Omega: d_{z} \mathcal{P}(Z)=T_{\mathcal{P}(z)} M, \operatorname{ker} d_{z} \mathcal{P}=\left(T_{\mathcal{P}(z)} M\right)^{\perp}$.

Regularity of the projection $\mathcal{P}$ is higher along $M$ itself; for example, for a $C^{2}$-submanifold the following improvement of regularity may be achieved: 
(4.2) Theorem. If $M$ is a $C^{2}$-submanifold, then

(i) for all $z \in M$, the mapping $\mathcal{P}$ is twice differentiable at $z$;

(ii) the mapping $M \ni z \mapsto d_{z}^{2} \mathcal{P} \in S_{2}(Z, Z)$ is continuous. (The symbol $S_{2}(Z, Z)$ stands for the Banach space of all symmetric bilinear operators $Z^{2} \rightarrow Z$.)

Despite the fact that $\left.\mathcal{P}\right|_{M}=\mathrm{id}_{M}$, the restriction to $M$ of the second derivative of $\mathcal{P}$ may have a non-trivial structure. For example, if $M$ is the unit sphere in $Z$, then

$$
\left(d_{z}^{2} \mathcal{P}\right)(\eta, \xi)=3(\eta \mid z)(\xi \mid z) z-(\eta \mid \xi) z-(\eta \mid z) \xi-(\xi \mid z) \eta
$$

for $z \in M$ and $\eta, \xi \in Z$.

Theorem (4.2) cannot be generalized to any neighbourhood of $M$ :

(4.3) Example. The curve $M:=\{(t, 0): t<0\} \cup\left\{\left(t, \frac{1}{3} t^{3}\right): t \geq 0\right\}$ is a $C^{2}$-submanifold of $Z=\mathbb{R}^{2}$. The projection $\mathcal{P}$ is not twice differentiable in any neighbourhood of $(0,0)$.

(4.4) Proposition. Let

$$
\varrho(x):=\varrho(x, M) \quad(=|x-\mathcal{P}(x)| \text { for } x \in \operatorname{dom} \mathcal{P}) .
$$

If $M$ is of class $C^{2}$, then

$$
\nabla_{x} \varrho=\frac{x-\mathcal{P}(x)}{|x-\mathcal{P}(x)|} \quad \text { for any } x \in \Omega \backslash M .
$$

This fact and Theorem (4.1) immediately give the following

(4.5) Corollary. The function $\varrho$ is of class $C^{k}$ in $\Omega \backslash M$ for $M$ being a submanifold of class $C^{k}(k \in\{2,3, \ldots, \infty, \omega\})$.

One of the first formulations of differential properties of $\varrho$ was presented in Serrin [16] (Chapter I, Lemma 3.1) in the following form:

If a hypersurface $M$ (i.e. a submanifold in $Z$ of codimension 1$)$ is of class $C^{3}$, then there is an open neighbourhood $\mathcal{O}$ of $M$ such that $\left.\varrho\right|_{\mathcal{O} \backslash M}$ is of class $C^{2}$.

Later on Gilbarg and Trudinger proved the following local version of Corollary (4.5) in the Appendix to [6]:

The restriction $\left.\varrho\right|_{\mathcal{O} \backslash M}$ is of class $C^{k}$ for some neighbourhood $\mathcal{O}$ of a $C^{k}$-hypersurface $M$ for $k=2,3, \ldots, \infty, \omega$.

Obviously, the continuous function $\varrho$ is not differentiable at any point of $M$. However, in the case of $M$ being a hypersurface one can smooth $\varrho$ by one-sided change of its sign: 
(4.6) Theorem (see Krantz-Parks [12]). Assume that the boundary $M$ of some set $G \in \operatorname{top} Z$ is a compact $C^{k}$-submanifold $(k \in\{1,2, \ldots, \infty, \omega\})$. Suppose also that $M \subset \Omega$ (this assumption is relevant only for $k=1$ ). Consider the signed distance function to $M$ :

$$
\delta(x):= \begin{cases}\varrho(x) & \text { if } x \in G \\ -\varrho(x) & \text { if } x \in Z \backslash G .\end{cases}
$$

Then $\delta$ is of class $C^{k}$ in some neighbourhood of $M$.

5. When is the whole space the domain of an orthogonal projection? If $\operatorname{dom} \mathcal{P}=Z$ then $M$ is called a Chebyshev set.

(5.1) TheOrem. Every non-empty closed and convex subset of $Z$ is a Chebyshev set.

The above theorem also holds for an infinite-dimensional Hilbert space (see Rudin [15], Theorem 4.10). Moreover, in finite-dimensional spaces also the converse theorem holds:

(5.2) Theorem (see Bunt [2], Motzkin [14]). If $\operatorname{dom} \mathcal{P}=Z$, then $M$ is non-empty, closed and convex.

A bounded Chebyshev set cannot be a submanifold of $Z$ :

(5.3) Theorem. Assume that $M$ is a $C^{2}$-submanifold of a Euclidean space $Z$ and $\operatorname{dom} \mathcal{P}=Z$. Then $M$ is an affine subspace of $Z$.

Under some additional assumptions the implication (5.2) is valid also for infinite-dimensional Hilbert spaces or even Banach spaces (see EfimovStechkin [4], Klee [10], Asplund [1]). Klee in [11] conjectures that in some (possibly non-separable) Hilbert spaces there exist non-convex Chebyshev sets. The question of existence of "Klee caverns" is also considered in Asplund [1].

6. Domains of projections onto graphs of some elementary functions. Now we illustrate the above theory with examples of orthogonal projections onto graphs of some numerical functions. In this section $Z=\mathbb{R}^{2}$.

(6.1) Example. The set $M:=\left\{\left(x, x^{2}\right): x \in \mathbb{R}\right\}$ is a closed analytic submanifold of $Z$. The domain of the orthogonal projection onto $M$ is $\operatorname{dom} \mathcal{P}=\mathbb{R}^{2} \backslash\{(0, t): t>1 / 2\}$ (see (3.14) and Fig. 2).

(6.2) Example. If we take $M:=\left\{\left(x, x^{2}\right): x \geq 0\right\}$ (clearly, $M$ is not a submanifold but it is closed), then $\operatorname{dom} \mathcal{P}=\mathbb{R}^{2} \backslash\{(x, y): x<0, y=$ $\left.\frac{3}{2}(-x)^{2 / 3}+\frac{1}{2}\right\}$ (see (2.4) and Fig. 3). 


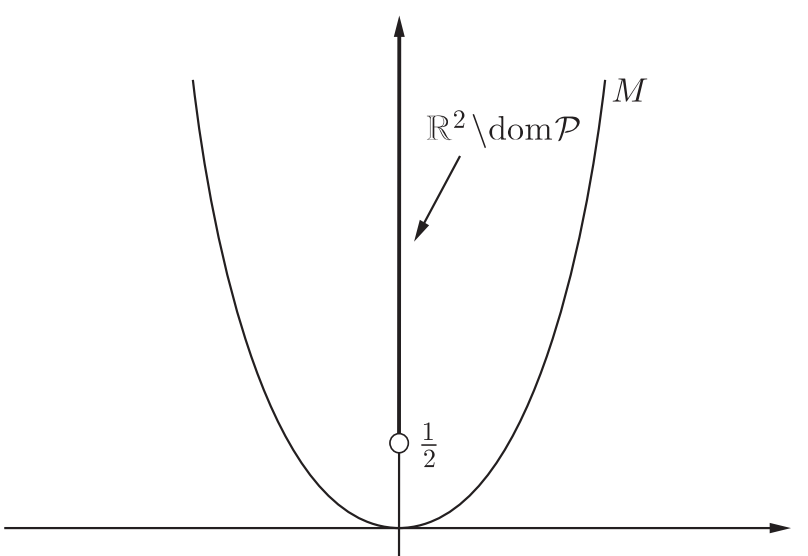

Fig. 2

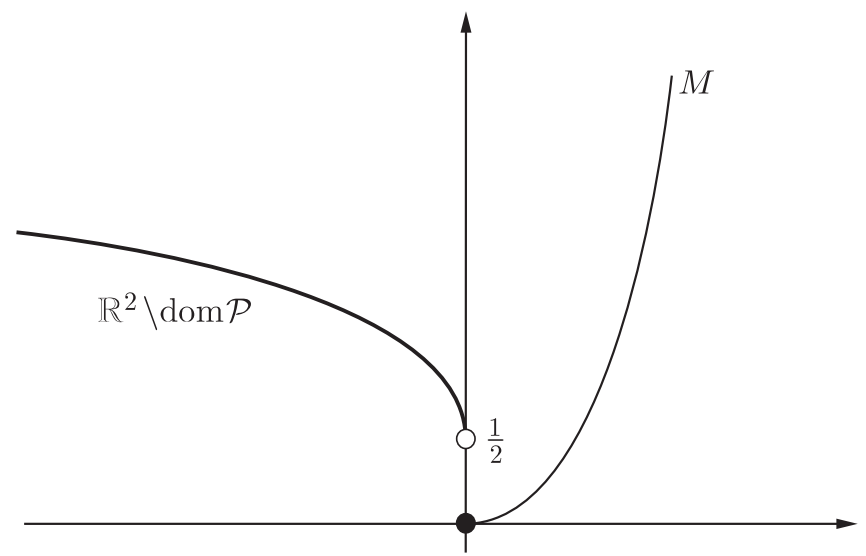

Fig. 3

(6.3) Example. Removing the point $(0,0)$ from the set $M$ of the preceding example we obtain a non-closed submanifold $M^{\prime}$ with

$$
\operatorname{dom} \mathcal{P}=\mathbb{R}^{2} \backslash\left(\left\{\left(0, \frac{1}{2}\right)\right\} \cup\left\{(x, y): x \leq 0, y<\frac{3}{2}(-x)^{2 / 3}+\frac{1}{2}\right\}\right)
$$

(see (3.14) and Fig. 4).

(6.4) ExAmPle. Let $M$ be the graph of $\mathbb{R} \ni x \mapsto \cos x$. Then $\operatorname{dom} \mathcal{P}=$ $\mathbb{R}^{2} \backslash \bigcup_{k \in \mathbb{Z}}\left\{(k \pi, y):(-1)^{k+1} y>0\right\}$ (see (3.14) and Fig. 5).

(6.5) ExAmple. We did not manage to find the exact shape of the domain of the orthogonal projection onto $M:=\left\{\left(x, e^{x}\right): x \in \mathbb{R}\right\}$ (naturally, 


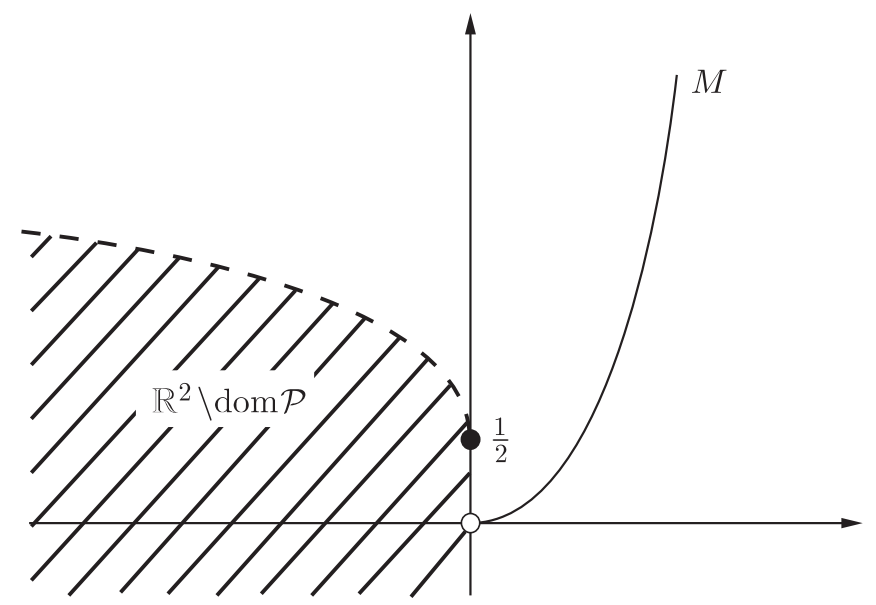

Fig. 4

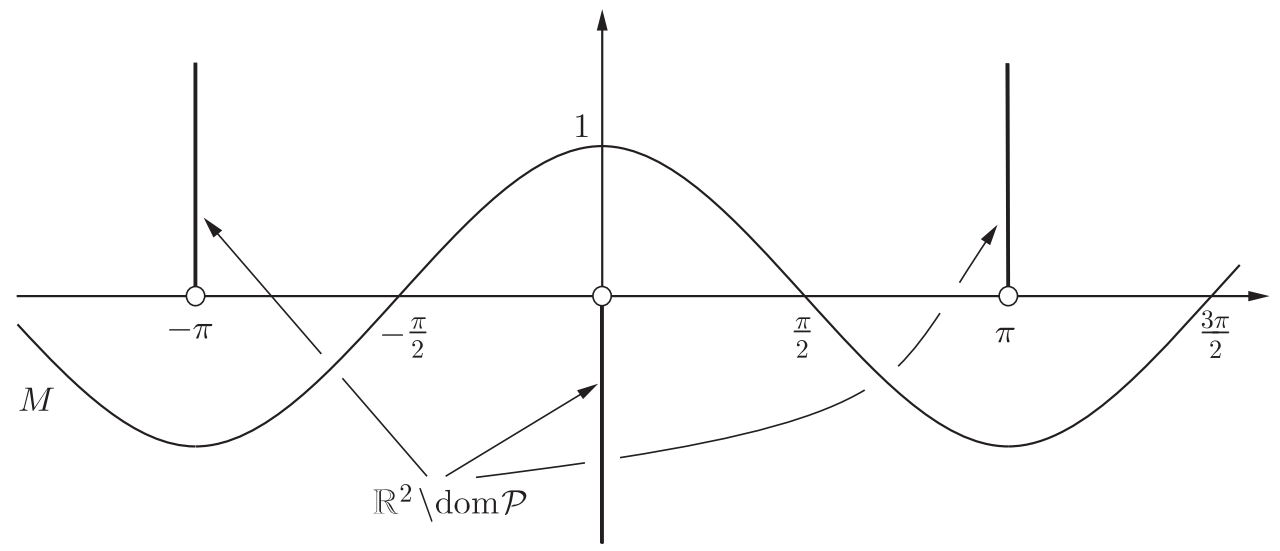

Fig. 5

$M$ is a closed analytic submanifold). The relation

$$
\begin{aligned}
& \mathbb{R}^{2} \backslash \operatorname{dom} \mathcal{P} \\
& \quad \subset\left\{(x, y): y>2 \sqrt{2},-\ln \sqrt{2 e}-u_{2}(y / 2 \sqrt{2})<x<-\ln \sqrt{2 e}-u_{1}(y / 2 \sqrt{2})\right\},
\end{aligned}
$$

where

$$
\begin{aligned}
& u_{1}(z):=z\left(z-\sqrt{z^{2}-1}\right)+\ln \left(z+\sqrt{z^{2}-1}\right) \\
& u_{2}(z):=z\left(z+\sqrt{z^{2}-1}\right)-\ln \left(z+\sqrt{z^{2}-1}\right) \quad \text { for all } z>1
\end{aligned}
$$

is all we know about $\operatorname{dom} \mathcal{P}$ (see (3.14) and (5.3); Fig. 6). 


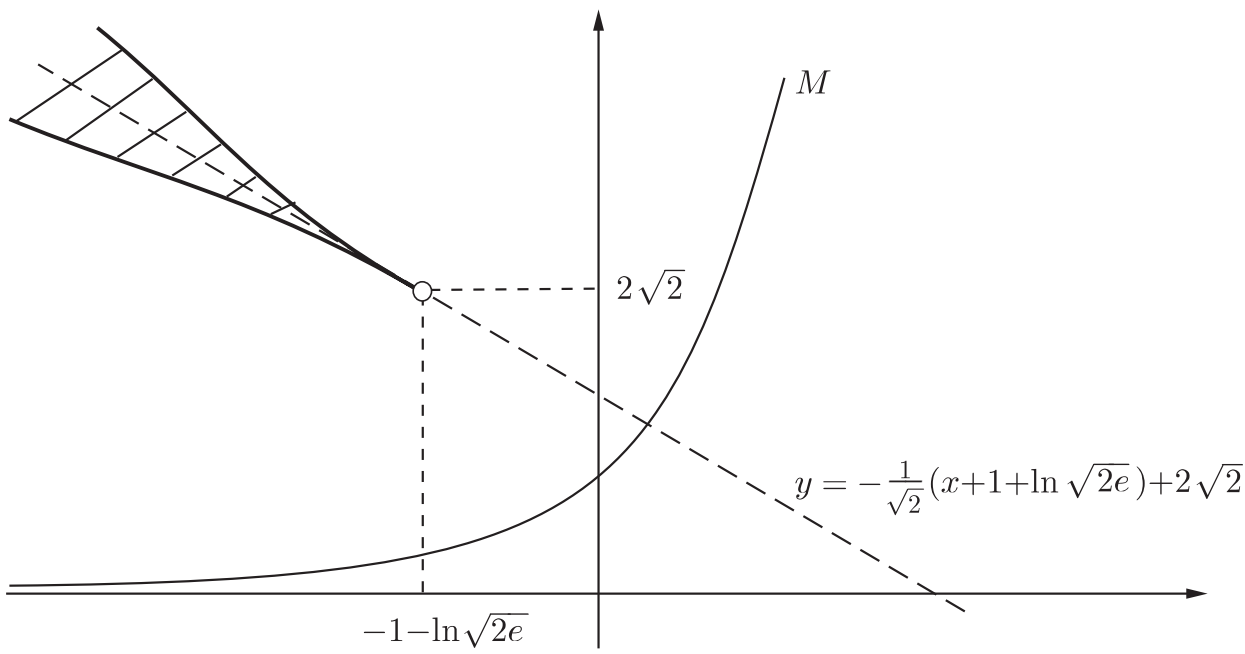

Fig. 6

\section{$1^{\prime}$. Proofs}

$\left(1^{\prime} .1\right)$ Lemma. If $a \in \Omega$ and $V$ is a neighbourhood of $\mathcal{P}(a)$ in $M$, then $\varrho(a, M \backslash V)>\varrho(a, M)$.

Pro of. If the inequality were inverse, then $\varrho(a, M \backslash V)=\varrho(a, M)$. There is a sequence $\left(x_{\nu}\right)_{\nu=1}^{\infty} \in(M \backslash V)^{\mathbb{N}}$ for which $\left|a-x_{\nu}\right| \rightarrow \varrho(a, M \backslash V)=\varrho(a, M)$ as $\nu \rightarrow \infty$. Since $\left|x_{\nu}\right| \leq\left|x_{\nu}-a\right|+|a|(\forall \nu)$, there are also an infinite set $A \subset \mathbb{N}$ and $b \in Z$ such that $x_{\nu} \rightarrow b$ as $A \ni \nu \rightarrow \infty$. Clearly, $|a-b|=\varrho(a, M)$, $b \neq \mathcal{P}(a), b \notin M$ and $b \neq a$. Choosing $z \in] a, b] \cap \operatorname{dom} \mathcal{P}(\neq \emptyset)$ we obtain

$$
b-z=\frac{|b-z|}{|z-a|}(z-a)
$$

and $|a-b| \leq|a-\mathcal{P}(a)| \leq|a-z|+|z-\mathcal{P}(z)| \leq|a-z|+|z-b|=|b-a|$. This means that for $u:=z-a$ and $v:=\mathcal{P}(z)-z$ we have $|u|+|v|=|u+v|$ and $|v|=|z-b|$, i.e. $v=\frac{|v|}{|u|} u$. Therefore, $b-z=\frac{|v|}{|u|} u=\mathcal{P}(z)-z$, contrary to $b \notin M$.

Proof of Theorem (1.3). Suppose that $\mathcal{P}$ is not continuous at $a \in \Omega$. There is a neighbourhood of $\mathcal{P}(a)$ on $M$ and a sequence $\left(a_{\nu}\right) \in(\operatorname{dom} \mathcal{P})^{\mathbb{N}}$ with $a_{\nu} \rightarrow a$ as $\nu \rightarrow \infty$ and $\mathcal{P}\left(a_{\nu}\right) \notin V$ for all $\nu$. Then

$$
\begin{aligned}
\forall \nu \in \mathbb{N}: \quad \varrho(a, M \backslash V) & \leq\left|a-\mathcal{P}\left(a_{\nu}\right)\right| \leq\left|a-a_{\nu}\right|+\left|a_{\nu}-\mathcal{P}\left(a_{\nu}\right)\right| \\
& =\left|a-a_{\nu}\right|+\varrho\left(a_{\nu}, M\right) .
\end{aligned}
$$

As a consequence, $\varrho(a, M \backslash V) \leq \varrho(a, M)$, which contradicts Lemma (1'.1). 
$\left(1^{\prime} .2\right)$ Lemma. For $\left.\left.t \in\right] 0,1\right]$ define $f_{t}: \operatorname{dom} \mathcal{P} \rightarrow Z$ by $f_{t}(z):=\mathcal{P}(z)+$ $t(z-\mathcal{P}(z))$. Then $f_{t}$ is an injection.

Proof. The case $t=1$ is easy. For $t \in] 0,1[$ suppose $a, b \in \operatorname{dom} \mathcal{P}$ satisfy $f_{t}(a)=f_{t}(b)$. By Remark (1.7) we can assume $f_{t}(a)=f_{t}(b)=0$ without loss of generality. So, for $s:=-t /(1-t)$ we have

$$
a^{\prime}:=\mathcal{P}(a)=s a, \quad b^{\prime}:=\mathcal{P}(b)=s b .
$$

Assume that $|a| \geq|b|$ and $|a|>0$. If $a, b$ were linearly dependent, then we would get $b \in[0, a]$ (if not, there is $\xi \in]-1,0[$ for which $b=\xi a$; this yields $\left.b^{\prime}=s \xi a \in\right] 0, \infty\left[\cdot a\right.$ and $\varrho(a, M) \leq\left|a-b^{\prime}\right|=|1-s \xi| \cdot|a|<$ $\left.(1+|s| \cdot|\xi|)|a|<(1-s)|a|=\left|a-a^{\prime}\right|=\varrho(a, M)\right)$. Therefore $b \in[0, a] \subset\left[a^{\prime}, a\right]$. By Theorem (1.5)(i), $b^{\prime}=\mathcal{P}(b)=a^{\prime}$. Thus $a=b$.

So it suffices to establish linear dependence of $a$ and $b$. Suppose not, i.e. $(a \mid b)<|a| \cdot|b| \leq|a|^{2}$. It follows that

$$
|a|^{2}-|b|^{2} \geq 0>2\left(a+\frac{(a \mid a-b)}{t|a-b|^{2}}(b-a) \mid a-b\right)
$$

and for

$$
x:=s\left(a+\frac{(a \mid a-b)}{t|a-b|^{2}}(b-a)\right)
$$

we get $\left|x-a^{\prime}\right|^{2}>\left|x-b^{\prime}\right|^{2}$. We have $((1-t)(a-x) \mid a-b)=0$, so $a-x \perp b-a$. Moreover, $a-x \perp x-a^{\prime}$ and $a-x \perp x-b^{\prime}$, because $x-a^{\prime}, x-b^{\prime} \in \mathbb{R} \cdot(b-a)$. By Pythagoras' Theorem,

$$
\begin{aligned}
\varrho(a, M)^{2} & \leq\left|a-b^{\prime}\right|^{2}=|a-x|^{2}+\left|x-b^{\prime}\right|^{2}<|a-x|^{2}+\left|x-a^{\prime}\right|^{2} \\
& =\left|a-a^{\prime}\right|^{2}=\varrho(a, M)^{2} .
\end{aligned}
$$

This contradiction completes the proof.

Proof of Theorem (1.5). The indirect proof of the fact that $\left|z-a^{\prime}\right|<$ $|z-x|$ for all $x \in M \backslash\left\{a^{\prime}\right\}$ uses the method of proof of Lemma (1'.1).

To show (ii), fix $a \in \Omega$ and a point $\left(\left[a, \mathcal{P}(a)[\ni) z_{0}:=\mathcal{P}(a)+t(a-\mathcal{P}(a))\right.\right.$ (for some $t \in] 0,1]$ ). The Brouwer theorem on the invariance of domain applied to the continuous injection $f_{t}: \Omega \ni z \mapsto \mathcal{P}(z)+t(z-\mathcal{P}(z)) \in$ $[z, \mathcal{P}(z)] \subset \operatorname{dom} \mathcal{P}$ completes the proof.

Proof of Corollary (1.6). Fix $a \in \mathcal{O}$ and suppose that $|b-a|=$ $\varrho(a, M)$ for some $b \in M \backslash\{f(a)\}$. It is clear that $a \neq b$. In view of Theorem (1.5), $] a, b] \subset \operatorname{dom} \mathcal{P}$ and $\mathcal{P}(z)=b$ for all $z \in] a, b]$. So, there is a sequence $\left.\left.\left(z_{\nu}\right) \in(] a, b\right] \cap(\operatorname{dom} \mathcal{P}) \cap \mathcal{O}\right)^{\mathbb{N}}$ convergent to $a$. Of course, $b=\mathcal{P}\left(z_{\nu}\right)=f\left(z_{\nu}\right)$ for all $\nu$. Since $f$ is continuous, we obtain the equality $b=f(a)$ contradicting the choice of $b$. 


\section{2'. Proofs}

Proof of Fact (2.2) is easy.

Proof of Fact (2.3). Fix $a \in D$. Then there is $r>\varrho(a, M)=\vartheta r$ (for some $\vartheta \in[0,1[)$ such that $M \cap \bar{B}(a, r) \in \operatorname{cotop} Z$. The inclusion $B(a,(1-\vartheta) r / 2) \subset D$ holds, because for $\widetilde{a} \in B(a,(1-\vartheta) r / 2)$ we have $\varrho(\widetilde{a}, M)<(1+\vartheta) r / 2, \bar{B}(\widetilde{a},(1+\vartheta) r / 2) \subset \bar{B}(a, r)$ and $\bar{B}(\widetilde{a},(1+\vartheta) r / 2) \cap M \in$ cotop $Z$.

Proof of Remark (2.4) rests on the simple

$\left(2^{\prime} .1\right)$ Remark. If $\emptyset \neq \mathcal{O} \subset D$ is open, then $\mathcal{O} \cap \operatorname{dom} \mathcal{P}$ is dense in $\mathcal{O}$.

Pr o o f. Consider $G \in(\operatorname{top} \mathcal{O}) \backslash\{\emptyset\}$ and $a \in G(\subset D)$. There is $a^{\prime} \in M$ for which $\left|a-a^{\prime}\right|=\varrho(a, M)$. Thus $\left.] a, a^{\prime}\right] \subset \operatorname{dom} \mathcal{P}$. Clearly, $\left.\left.G \cap\right] a, a^{\prime}\right] \neq \emptyset$, and consequently $G \cap \mathcal{O} \cap \operatorname{dom} \mathcal{P} \neq \emptyset$.

$\left(2^{\prime} .2\right)$ Lemma. If $a \in D \cap \operatorname{dom} \mathcal{P}$ and $V$ is a neighbourhood of $\mathcal{P}(a)$ in $M$, then $\varrho(a, M \backslash V)>\varrho(a, M)$.

Proof. For an indirect proof suppose that $\varrho(a, M \backslash V)=\varrho(a, M)$. There is a sequence $\left(x_{\nu}\right) \in(M \backslash V)^{\mathbb{N}}$ for which $\left|a-x_{\nu}\right| \rightarrow \varrho(a, M)$ as $\nu \rightarrow \infty$. It is bounded, so there is an infinite set $A \subset \mathbb{N}$ and $b \in Z \backslash\{\mathcal{P}(a)\}$ such that $x_{\nu} \rightarrow b$ as $A \ni \nu \rightarrow \infty$. Obviously, $|a-b|=\varrho(a, M)$. There is a radius $r>\varrho(a, M)$ such that $M \cap \bar{B}(a, r) \in \operatorname{cotop} Z$, so we have $x_{\nu} \in \bar{B}(a, r) \cap M$ (for almost all $\nu \in \mathbb{N}$ ). Hence, $b \in \bar{B}(a, r) \cap M \subset M$ and $b \neq \mathcal{P}(a)$, which contradicts $|a-b|=\varrho(a, M)$.

Proof of Remark (2.5) is analogous to the one of Theorem (1.3).

Proof of Proposition (2.6). For a given point $a \in \Omega$ there is a closed neighbourhood $F$ of $\mathcal{P}(a)$ in $M$. By Lemma (1'.1) there exists $r$ with $\varrho(a, M)<r<\varrho(a, M \backslash F)$. Clearly, $M \cap \bar{B}(a, r)=F \cap \bar{B}(a, r)$.

Proof of Theorem (2.7). Suppose $a \neq a^{\prime}$. Without loss of generality we can assume $a=0$. According to Theorem (1.5), ]0, $\left.a^{\prime}\right] \subset \operatorname{dom} \mathcal{P}$ and $\mathcal{P}(z)=a^{\prime}$ for all $\left.\left.z \in\right] 0, a^{\prime}\right]$. Fix $\left.\left.z \in\right] 0, a^{\prime}\right]$ and set $d:=\varrho(z, M)=\left|z-a^{\prime}\right|<$ $\left|a^{\prime}\right|=: r$. There is $0<\delta<\sqrt{r(r+d)}$ for which $M \cap \bar{B}\left(a^{\prime}, \delta\right) \in \operatorname{cotop} Z$. We have

$$
\varrho(z, M)<\sqrt{d^{2}+\delta^{2} \frac{r-d}{r}}=: r(z)(<r) .
$$

To show that $\bar{B}(z, r(z)) \cap M \in \operatorname{cotop} Z$ (which will complete the proof) it suffices to prove that $\bar{B}(z, r(z)) \subset B(0, r) \cup \bar{B}\left(a^{\prime}, \delta\right)$. Indeed, let $x \in$ $\bar{B}(z, r(z))$ with $|x| \geq r$. Then $(x \mid z)>0$ and consequently $\left(x \mid a^{\prime}\right)>0$. If

$$
x^{\prime}:=\frac{\left(x \mid a^{\prime}\right)}{\left|a^{\prime}\right|^{2}} a^{\prime}
$$


denotes the orthogonal projection of $x$ onto $\mathbb{R} a^{\prime}$, then

$$
\left|x^{\prime}\right|=\frac{|x|^{2}+r^{2}-\left|x-a^{\prime}\right|^{2}}{2 r} .
$$

Further, $\left|x^{\prime}-z\right|=|| x^{\prime}|-(r-d)|$, since $x^{\prime}, z \in \mathbb{R}_{+} a^{\prime}$. Hence

$$
\begin{aligned}
r(z)^{2} & \geq|x-z|^{2}=\left|x-x^{\prime}\right|^{2}+\left|x^{\prime}-z\right|^{2} \\
& =\left(|x|^{2}-\left|x^{\prime}\right|^{2}\right)+\left|x^{\prime}-z\right|^{2} \geq \frac{r-d}{r}\left|x-a^{\prime}\right|^{2}+d^{2},
\end{aligned}
$$

which means that indeed $\delta \geq\left|x-a^{\prime}\right|$.

Proof of Theorem (2.8). Define

$$
f_{t}: \operatorname{dom} \mathcal{P} \ni z \mapsto \mathcal{P}(z)+t(z-\mathcal{P}(z)) \in Z .
$$

It is an injection with values in dom $\mathcal{P}$ (by Lemma $\left(1^{\prime} .2\right)$ and Theorem (1.5)). Moreover, $\mathcal{P} \circ f_{t}=\mathcal{P}, f_{t}(\Omega) \subset \Omega$ and $f_{t}^{-1}(w)=\frac{1}{t}(w-(1-t) \mathcal{P}(w))$.

(i) $\Rightarrow$ (ii). In view of the Brouwer theorem on invariance of domain, $f_{t}(\Omega)$ is a neighbourhood of $f_{t}(a)=\mathcal{P}(a)+t(a-\mathcal{P}(a))$. Clearly, $t f_{t}^{-1}$ is an injection on it.

(ii) $\Rightarrow($ i). According to the assumptions $a \in D \cap \operatorname{dom} \mathcal{P}$. There is an open neighbourhood $U \subset \Omega$ of $\widetilde{a}:=f_{t}(a)$ such that the mapping $Q: U \ni w \mapsto$ $\frac{1}{t}(w-(1-t) \mathcal{P}(w)) \in Z$ is a continuous injection (see Theorem (1.3)). By the Brouwer theorem, $Q(U) \in \operatorname{top} Z$ and $Q: U \rightarrow Q(U)$ is a homeomorphism. Also, $Q^{-1}(z)=f_{t}(z)$ and $\left(\mathcal{P} \circ Q^{-1}\right)(z)=\mathcal{P}(z)$ for any $z \in f_{t}^{-1}(U)(\subset Q(U))$. The map $\left.f_{t}\right|_{D}$ is continuous by Remark (2.5), hence there is $\mathcal{O} \in$ top $Z$ for which $\left(\left.f_{t}\right|_{D}\right)^{-1}(U)=\mathcal{O} \cap D \cap \operatorname{dom} \mathcal{P}$. By $(2.3), D_{0}:=\mathcal{O} \cap D \cap Q(U)$ is an open neighbourhood of $a$. It remains to show that $D_{0} \subset \operatorname{dom} \mathcal{P}$. For $z \in D_{0}$, Remark $\left(2^{\prime} .1\right)$ enables us to choose a sequence $\left(z_{\nu}\right) \in\left(D_{0} \cap \operatorname{dom} \mathcal{P}\right)^{\mathbb{N}}$ convergent to $z$. Then

$$
\left|z-\left(\mathcal{P} \circ Q^{-1}\right)(z)\right| \underset{\nu \rightarrow \infty}{\longleftarrow}\left|z_{\nu}-\left(\mathcal{P} \circ Q^{-1}\right)\left(z_{\nu}\right)\right|=\left|z_{\nu}-\mathcal{P}\left(z_{\nu}\right)\right| \underset{\nu \rightarrow \infty}{\longrightarrow} \varrho(z, M) .
$$

Consequently, $\forall z \in D_{0}:\left|z-\left(\mathcal{P} \circ Q^{-1}\right)(z)\right|=\varrho(z, M)$. From Corollary (1.6) it follows directly that $D_{0} \subset \operatorname{dom} \mathcal{P}$, which completes the proof.

\section{$3^{\prime}$. Proofs}

Proof of Remark (3.1) is based on the necessary condition for a local minimum of a differentiable function. then

$\left(3^{\prime} .1\right)$ Lemma. If $M \subset Z$ is a submanifold and $F \subset M$ a compact set, $\sup \left\{\varrho\left(\frac{z-y}{|z-y|}, T_{y} M\right): z \in M, y \in F, 0<|z-y|<\delta\right\} \rightarrow 0 \quad$ as $\delta \rightarrow 0$. 
Proof. It is sufficient to show that this condition holds locally on $M$, i.e. $\forall a \in M \exists \mathcal{O} \in \operatorname{top} M: \mathcal{O}$ is a neighbourhood of $a$ and

$$
\sup \left\{\varrho\left(\frac{z-y}{|z-y|}, T_{y} M\right): y, z \in \mathcal{O}, 0<|z-y|<\delta\right\} \rightarrow 0 \quad \text { as } \delta \rightarrow 0 .
$$

Fix $a \in M$ and an inverse chart $f: H \leftrightarrow M$ for which $f\left(a_{0}\right)=a$ (where $H$ is a linear subspace of a Euclidean space $X$, with $\operatorname{dim} X=\operatorname{dim} Z$; $\rightarrow$ denotes a partial mapping). We can assume, diminishing the domain if necessary, that $f^{-1}$ satisfies the Lipschitz condition on its domain. For a fixed convex compact neighbourhood $V \subset \operatorname{dom} f$ of $a_{0}$, the set $\mathcal{O}:=f(V)$ will prove to be the suitable neighbourhood of $a$. The mapping

$$
\alpha:] 0, \infty\left[\ni \delta \mapsto \sup \left\{\left|f^{\prime}(u)-f^{\prime}(v)\right|: u, v \in V,|u-v| \leq \delta\right\}\right.
$$

is increasing and $\lim _{\delta \rightarrow 0} \alpha(\delta)=0$. Define $\vartheta:=\inf _{h \in V} \min \left\{\left|d_{h} f(\xi)\right|:|\xi|=1\right\}$ $\left(=\left|d_{h_{1}} f\left(\xi_{0}\right)\right|>0\right.$ for some $h_{1} \in V$ and $\left.\xi_{0} \in\{z:|z|=1\}\right)$. Let $L$ be the Lipschitz constant for $f^{-1}$. Fix $\left.\left.\varepsilon \in\right] 0,1\right]$ and $r>0$ for which $\alpha(r) \leq \varepsilon \vartheta / 2$. For $\delta \in] 0, r / L\left[\right.$ and $z, z_{0} \in f(V)$ with $0<\left|z-z_{0}\right| \leq \delta$, set $h_{0}:=f^{-1}\left(z_{0}\right)$, $h:=f^{-1}(z)$. We have $\left|h-h_{0}\right| \leq r$ and, by the Lagrange theorem,

$$
\left|f(h)-f\left(h_{0}\right)-d_{h_{0}} f\left(h-h_{0}\right)\right| \leq \alpha(r)\left|h-h_{0}\right| \leq\left|h-h_{0}\right| \frac{\varepsilon \vartheta}{2} .
$$

In particular, for $\varepsilon=1$ we obtain

$$
\frac{\vartheta}{2} \geq\left|\frac{f(h)-f\left(h_{0}\right)}{\left|h-h_{0}\right|}-d_{h_{0}} f\left(\frac{h-h_{0}}{\left|h-h_{0}\right|}\right)\right| \geq \vartheta-\frac{\left|f(h)-f\left(h_{0}\right)\right|}{\left|h-h_{0}\right|}
$$

and consequently

$$
\frac{\left|h-h_{0}\right|}{\left|f(h)-f\left(h_{0}\right)\right|} \leq \frac{2}{\vartheta} .
$$

Going back to the general case of $\varepsilon>0$ and noticing that

$$
\begin{aligned}
\frac{z-z_{0}}{\left|z-z_{0}\right|}= & \frac{\left|h-h_{0}\right|}{\left|f(h)-f\left(h_{0}\right)\right|} d_{h_{0}} f\left(\frac{h-h_{0}}{\left|f(h)-f\left(h_{0}\right)\right|}\right) \\
& +\frac{\left|h-h_{0}\right|}{\left|f(h)-f\left(h_{0}\right)\right|}\left(\frac{f(h)-f\left(h_{0}\right)}{\left|h-h_{0}\right|}-d_{h_{0}} f\left(\frac{h-h_{0}}{\left|f(h)-f\left(h_{0}\right)\right|}\right)\right)
\end{aligned}
$$

(the first component is in $T_{z_{0}} M$ ) we come to the desired conclusion:

$$
\begin{aligned}
\varrho\left(\frac{z-z_{0}}{\left|z-z_{0}\right|}, T_{z_{0}} M\right) \\
\leq \frac{\left|h-h_{0}\right|}{\left|f(h)-f\left(h_{0}\right)\right|}\left(\frac{f(h)-f\left(h_{0}\right)}{\left|h-h_{0}\right|}-d_{h_{0}} f\left(\frac{h-h_{0}}{\left|f(h)-f\left(h_{0}\right)\right|}\right)\right) \\
\leq \frac{2}{\vartheta} \cdot \frac{\varepsilon \cdot \vartheta}{2}=\varepsilon .
\end{aligned}
$$


Reasoning by reductio ad absurdum it is easy to derive from Lemma $\left(3^{\prime} .1\right)$ the following

$\left(3^{\prime} .2\right)$ Lemma. If $M \subset Z$ is a submanifold and $F \subset M$ is compact, then $\exists r>0 \forall z \in F: M \cap\left(\bar{K}_{z}(r) \backslash\{z\}\right)=\emptyset$.

$\left(3^{\prime} .3\right)$ Lemma. Let $S:=\{z \in Z:|z|=1\}$. Fix subspaces $X, Y$ in $Z$. Denote by $d(X, Y)$ their distance in the Hausdorff metric (see (3.4)). For $\emptyset \neq A, B \subset Z$ put $\varrho(A, B):=\inf \{|a-b|: a \in A, b \in B\}$. Consider $R, \eta>0$ for which $\operatorname{Rd}(X, Y)<\eta$ and a point $a \in B_{R} \cap X$, where $B_{R}:=\{z \in Z$ : $|z| \leq R\}$. Assume that $\varrho([-R y, R y], a+[-R x, R x]) \geq \eta$ whenever $y \in S \cap Y$, $x \in S \cap X$ and $|x-y| \leq d(X, Y)$. Then $\left(B_{R} \cap Y\right) \cap\left(a+\left(B_{R} \cap X\right)\right)=\emptyset$.

Proof. Let $\Pi: Z \rightarrow Y$ stand for the linear orthogonal projection. There is $y \in S \cap Y$ such that $\Pi(a) \in[-R y, R y]$, and $x \in S \cap X$ for which $|x-y|=\varrho(y, S \cap X)(\leq d(X, Y))$. Hence $|a-\Pi(a)| \geq \eta$. Therefore $\left(B_{R} \cap\right.$ $Y) \cap\left(a+\left(B_{R} \cap X\right)\right)=\emptyset$, because if $a+t x \in Y \cap\left(a+\left(B_{R} \cap X\right)\right)$ (for some $|t| \leq R$ and $x \in S \cap X)$ then $\Pi(a+t x)=a+t x$ implies

$$
\eta \leq|\Pi(a)-a| \leq R|x-\Pi(x)| \leq R d(X, Y),
$$

which contradicts our assumptions.

Proof of Theorem (3.2). To prove (i) we only need to consider the case $\operatorname{dim} M<\operatorname{dim} Z$. Let $L>0$ be the Lipschitz constant for $T$ on $F$. Fix $\vartheta, c>0$ so that $\vartheta<c$ and $\vartheta+c<1$. From Lemma $\left(3^{\prime} .1\right)$ it follows that there is $\delta \in] 0, \vartheta / L]$ for which

$$
\sup \left\{\varrho\left(\frac{z-y}{|z-y|}, T_{y} M\right): y, z \in F, 0<|z-y|<\delta\right\} \leq \frac{1+(c+\vartheta)^{2}}{2(1+c+\vartheta)} .
$$

If we show that $K_{z_{1}}(\vartheta / L) \cap K_{z_{2}}(\vartheta / L)=\emptyset$ whenever $z_{1}, z_{2} \in F$ and $0<$ $\left|z_{1}-z_{2}\right| \leq \delta$, then $r:=\delta / 2$ will suit the assertion.

First, we put $S:=\{z \in Z:|z|=1\}$ and prove the auxiliary fact:

$$
\begin{aligned}
& \varrho\left(z_{1}+\frac{\vartheta}{L}\left[-\zeta_{1}, \zeta_{1}\right], z_{2}+\frac{\vartheta}{L}\left[-\zeta_{2}, \zeta_{2}\right]\right) \geq c\left|z_{1}-z_{2}\right| \text { whenever } z_{1}, z_{2} \in \\
& F, 0<\left|z_{1}-z_{2}\right|<\delta, \zeta_{i} \in S \cap T_{z_{i}}^{\perp}(i=1,2) \text { and }\left|\zeta_{1}-\zeta_{2}\right| \leq d\left(T_{z_{1}}^{\perp}, T_{z_{2}}^{\perp}\right)
\end{aligned}
$$

$$
\text { (of course, } T_{z}^{\perp}:=\left(T_{z} M\right)^{\perp} \text { ). }
$$

If $\left(3^{\prime} .4\right)$ were false we could find $t_{1}, t_{2} \in[-\vartheta / L, \vartheta / L]$ for which $\mid z_{1}+t_{1} \zeta_{1}-$ $z_{2}-t_{2} \zeta_{2}|<c| z_{1}-z_{2} \mid$ and hence $c\left|z_{1}-z_{2}\right|>\left|\left(z_{1}-z_{2}\right)+\left(t_{1}-t_{2}\right) \zeta_{2}\right|-t_{1}\left|\zeta_{1}-\zeta_{2}\right|$. The following inequalities hold:

$$
\left|t_{i}\left(\zeta_{1}-\zeta_{2}\right)\right| \leq \frac{\vartheta}{L} d\left(T_{z_{1}}^{\perp}, T_{z_{2}}^{\perp}\right)=\frac{\vartheta}{L} d\left(T_{z_{1}}, T_{z_{2}}\right) \leq \vartheta\left|z_{1}-z_{2}\right|
$$

(see (3.5)). Putting $s:=\left(t_{2}-t_{1}\right) /\left|z_{2}-z_{1}\right|$ we obtain

$$
\left|\frac{z_{2}-z_{1}}{\left|z_{1}-z_{2}\right|}+s \zeta_{2}\right|<c+\vartheta \quad \text { and } \quad|s| \leq c+\vartheta+1 .
$$


Combining these two inequalities we get

$$
\begin{aligned}
(c+\vartheta)^{2} & >1+s^{2}+2 s\left(\frac{z_{2}-z_{1}}{\left|z_{1}-z_{2}\right|} \mid \zeta_{2}\right) \geq 1-2|s| \cdot\left|\left(\frac{z_{2}-z_{1}}{\left|z_{1}-z_{2}\right|} \mid \zeta_{2}\right)\right| \\
& \geq 1-2(c+\vartheta+1) \varrho\left(\frac{z_{2}-z_{1}}{\left|z_{1}-z_{2}\right|}, T_{z_{2}}\right) \\
& \geq 1-2(c+\vartheta+1) \frac{1-(c+\vartheta)^{2}}{2(c+\vartheta+1)}=(c+\vartheta)^{2} .
\end{aligned}
$$

This contradiction proves $\left(3^{\prime} \cdot 4\right)$.

Now, to show (i) fix $z_{1}, z_{2} \in F$ such that $0<\left|z_{1}-z_{2}\right| \leq \delta$. We can apply Lemma $\left(3^{\prime} .3\right)$ to $X:=T_{z_{1}}^{\perp}, Y:=T_{z_{2}}^{\perp}, R:=\vartheta / L, \eta:=c\left|z_{1}-z_{2}\right|$ and $a:=z_{2}-z_{1}$, for we have

$$
d\left(T_{z_{1}}, T_{z_{2}}\right) \leq L\left|z_{1}-z_{2}\right|=\frac{c\left|z_{1}-z_{2}\right|}{\vartheta / L} \cdot \frac{\vartheta}{c}<\frac{\eta}{R},
$$

and in view of $\left(3^{\prime} .4\right)$ all the assumptions of Lemma $\left(3^{\prime} .3\right)$ hold. Thus indeed $K_{z_{1}}(\vartheta / L) \cap K_{z_{2}}(\vartheta / L)=\emptyset$.

To prove (ii) consider a compact set $\widetilde{F} \subset M$ for which $F \subset \operatorname{int}_{M} \widetilde{F}$. Fix $z_{0} \in F$ and a number $0<r<\varrho\left(z_{0}, M \backslash \operatorname{int}_{M} \widetilde{F}\right)$ satisfying (i). For $z \in K_{z_{0}}(r)$ and $\delta:=\left|z-z_{0}\right|(<r)$ we have $z_{0} \in E:=\bar{B}(z, \delta) \cap M=\bar{B}(z, \delta) \cap F$. Since $E$ is compact, there is $z_{1} \in E(\subset M)$ for which $\left|z_{1}-z\right|=\varrho(z, E)=\varrho(z, M)$. But $\left|z-z_{1}\right| \leq \delta<r$, thus, by Remark (3.1), $z \in K_{z_{1}}(r)\left(\cap K_{z_{0}}(r)\right)$ and consequently $z_{1}=z_{0}$.

EXAMPLE 3.7 is easy to analyze.

$\left(3^{\prime} .5\right)$ Lemma. Consider a submanifold $M \subset Z$ and a continuous function $r: M \rightarrow] 0, \infty\left[\right.$ such that for all $z \in M, K_{z}(r(z)) \subset \operatorname{dom} \mathcal{P}$ and $\left.\mathcal{P}\right|_{K_{z}(r(z))}$ $\equiv z$. Then $M \subset \bigcup_{z \in M} K_{z}(r(z)) \in \operatorname{top} Z$.

Proof. Fix $z_{0} \in M, x_{0} \in K_{z_{0}}\left(r\left(z_{0}\right)\right)$ and $\vartheta \in \mathbb{R}$ such that $\left|z_{0}-x_{0}\right|<\vartheta<$ $r\left(z_{0}\right)$. For a fixed compact neighbourhood $F \subset M$ of $z_{0}$ we put $V:=F \cap\{z \in$ $M: r(z) \geq \vartheta\}$. Compactness of $\partial V$ enables us to choose $y_{0} \in \partial V$ such that $\varrho\left(x_{0}, \partial V\right)=\left|x_{0}-y_{0}\right|\left(>\left|x_{0}-z_{0}\right|\right)$. We claim that $B\left(x_{0}, s\right) \subset \bigcup_{z \in M} K_{z}(r(z))$, where $s:=\min \left\{\delta / 2, \vartheta-\left|z_{0}-x_{0}\right|\right\}$ and $\delta:=\varrho\left(x_{0}, \partial V\right)-\left|z_{0}-x_{0}\right|$. Indeed, fix $x \in B\left(x_{0}, s\right)$ and $z \in V$ for which $|x-z|=\varrho(x, V)$. Then $z \in M^{\prime}:=\operatorname{int}_{M} V$, for otherwise, i.e. if $z \in \partial V$, we would have $\left|x-z_{0}\right|<-\delta / 2+\varrho\left(x_{0}, \partial V\right) \leq$ $\left|x_{0}-z\right|-\delta / 2<|x-z|$, contrary to the choice of $z$. Hence, by Remark (3.1), $x-z \perp T_{z} M^{\prime}=T_{z} M$. Moreover, $|x-z| \leq\left|x-x_{0}\right|+\left|x_{0}-z_{0}\right|<\vartheta-\mid z_{0}-$ $x_{0}|+| z_{0}-x_{0} \mid \leq r(z)$, so $x \in K_{z}(r(z))$.

$\left(3^{\prime} .6\right)$ Theorem. Consider a $C^{k}$-submanifold $M \subset Z(k \in\{1,2, \ldots, \infty\})$ satisfying condition (3.3). Then there exists a $C^{k}$-function $\left.r: M \rightarrow\right] 0, \infty[$ such that for all $z \in M, K_{z}(r(z)) \subset \operatorname{dom} \mathcal{P}$ and $\left.\mathcal{P}\right|_{K_{z}(r(z))} \equiv z$. Moreover, 


$$
\begin{aligned}
& \bigcup_{z \in M} K_{z}(r(z)) \in \operatorname{top} Z \text { and } \\
& \qquad \forall z, y \in M: \quad\left(z \neq y \Rightarrow K_{z}(r(z)) \cap K_{y}(r(y))=\emptyset\right) .
\end{aligned}
$$

Pr o of. There exists a family $\left\{F_{i}\right\}_{i=1}^{\infty}$ of compact subsets of $M$ such that $M=\bigcup_{i=1}^{\infty} F_{i}$ and $F_{i} \subset \operatorname{int}_{M} F_{i+1}(i=1,2, \ldots)$. Also there are $C^{k}$-functions $\lambda_{i}: M \rightarrow[0,1](i=1,2, \ldots)$ with $\left.\lambda_{i}\right|_{F_{i}} \equiv 1$ and $\operatorname{supp} \lambda_{i} \subset \operatorname{int}_{M} F_{i+1}(i=$ $1,2, \ldots)$. Put $\lambda_{0} \equiv 0$. By Theorem (3.2), for any $i \in\{1,2, \ldots\}$ there is $r_{i}>0$ such that for all $z \in F_{i+1}, K_{z}\left(r_{i}\right) \subset \operatorname{dom} \mathcal{P}$ and $\left.\mathcal{P}\right|_{K_{z}\left(r_{i}\right)} \equiv z$. Clearly, we can assume that $r_{1} \geq r_{2} \geq \ldots$ For a fixed $i \in\{1,2, \ldots\}$ define $h_{i}:\left(\operatorname{int}_{M} F_{i+1}\right) \backslash \operatorname{supp} \lambda_{i-1} \rightarrow \mathbb{R}$ by $h_{i}(z):=r_{i} \lambda_{i}(z)+r_{i+1}\left(1-\lambda_{i}(z)\right)$. Obviously, this is a $C^{k}$-function. If $i \neq j$ and $z \in\left(\operatorname{dom} h_{i}\right) \cap\left(\operatorname{dom} h_{j}\right)$, then $h_{i}(z)=h_{j}(z)$, so $r:=\bigcup_{i=1}^{\infty} h_{i} \subset M \times \mathbb{R}$ is a $C^{k}$-function on $M$. Moreover, for any $z \in \operatorname{dom} h_{i}$ we have $0<r(z) \leq r_{i}$, so $r: M \rightarrow \mathbb{R}$ satisfies the desired condition.

Proof of Theorem (3.8). Theorem (3'.6) ensures the existence of a continuous function $r: M \rightarrow] 0, \infty\left[\right.$ such that for all $x \in M, K_{x}(r(x)) \subset$ $\operatorname{dom} \mathcal{P}$ and $\left.\mathcal{P}\right|_{K_{x}(r(x))} \equiv x$. Fix $\left.\vartheta \in\right] 0, r(a)[$. There is $s>0$ for which $B(a, s) \cap M \subset\{x \in M: r(x)>\vartheta\}$. If $r_{0}:=\min \{s, \vartheta / 2\}$, then

$$
\mathcal{O}:=\bigcup_{x \in M \cap B\left(a, r_{0}\right)} K_{x}\left(r_{0}\right)=\bigcup_{x \in M \cap \mathcal{O}} K_{x}\left(r_{0}\right)
$$

is an open neighbourhood of $a$ in $Z$ (see $\left(3^{\prime} .5\right)$ ). For any $z \in \mathcal{O}$ there exists $x \in \mathcal{O} \cap M$ such that $z \in K_{x}\left(r_{0}\right)$, thus $\mathcal{O} \subset \operatorname{dom} \mathcal{P}$. The remaining assertion results from the fact that any two distinct elements of the family $\left\{K_{x}\left(r_{0}\right)\right\}_{x \in \mathcal{O} \cap M}$ are disjoint.

Proof of Corollary (3.9). Theorem (3.8) implies the existence of $V \in \operatorname{top} Z$ for which $\mathcal{P}(a) \in V \subset \operatorname{dom} \mathcal{P}$ and $\forall z \in V \forall x \in V \cap M:(z-x \perp$ $\left.T_{x} M \Rightarrow \mathcal{P}(z)=x\right)$. For some $\left.\lambda \in\right] 0,1[$ we have $\widetilde{a}:=\mathcal{P}(a)+\lambda(a-\mathcal{P}(a)) \in V$. The injection $f_{\lambda}$ : $\operatorname{dom} \mathcal{P} \ni z \mapsto \mathcal{P}(z)+\lambda(z-\mathcal{P}(z))$ is continuous (see (1.3) and (1'.2)); moreover, $f_{\lambda}(a) \in V \cap f_{\lambda}(\Omega) \in \operatorname{top} Z$. Hence, there exists $\mathcal{O} \in \operatorname{top} T_{\mathcal{P}(a)}^{\perp} M$ such that $0 \in \mathcal{O}$ and $\widetilde{a}+\lambda \mathcal{O} \subset V \cap f_{\lambda}(\Omega)$. Applying Theorem (3.8) to a fixed $u \in \mathcal{O}$ we obtain $\mathcal{P}(\widetilde{a}+\lambda u)=\mathcal{P}(a)$. Since $a+u=$ $f_{\lambda}^{-1}(\widetilde{a}+\lambda u) \in \Omega$, it follows that $\mathcal{P}(a+u)=\mathcal{P}(a)$, completing the proof.

Proof of Corollary (3.10). Theorem (3.8) lets us choose a set $V \in \operatorname{top} Z$ for which $\mathcal{P}(a) \in V \subset \operatorname{dom} \mathcal{P}$ and $\forall z \in V \forall x \in V \cap M:(z-x \perp$ $\left.T_{x} M \Rightarrow \mathcal{P}(z)=x\right)$. Assume that $\mathcal{P}(a) \neq a$ and fix $\left.\left.t \in\right] 0,1\right]$ such that $\widetilde{a}:=\mathcal{P}(a)+t(a-\mathcal{P}(a)) \in V$. Define $\phi_{t}: \mathcal{O} \ni z \mapsto \phi(z)+t(z-\phi(z)) \in Z$ and $G:=\phi_{t}^{-1}(V) \cap \phi^{-1}(V)$; obviously, $a \in G$. By the choice of $V$ we have $\mathcal{P} \circ \phi_{t}(z)=\phi(z)$ for any $z \in G$. In order to show injectivity of $\left.\phi_{t}\right|_{G}$ consider 
the map

$$
Q: \operatorname{dom} \mathcal{P} \ni v \mapsto \frac{1}{t}(v-(1-t) \mathcal{P}(v)) .
$$

For any $z \in G$ we have $Q\left(\phi_{t}(z)\right)=z$, so $\left.\phi_{t}\right|_{G}$ (and surely $\left.Q\right|_{\phi_{t}(G)}$ ) is an injection. Hence, by the Brouwer theorem, $\phi_{t}(G)$ is open. Therefore, $\widetilde{a} \in$ $\phi_{t}(G) \subset \Omega$, which together with injectivity of $\left.Q\right|_{\phi_{t}(G)}$ and Theorem (2.8) means that $a \in \Omega$.

To show that $\phi=\mathcal{P}$ in some neighbourhood of $a$-and end the proof in this way - it suffices to prove that $f_{t}=\phi_{t}$ in this neighbourhood, where $f_{t}: \operatorname{dom} \mathcal{P} \ni z \mapsto \mathcal{P}(z)+t(z-\mathcal{P}(z))$. Indeed, for every $z$ from the open set $G_{0}:=G \cap\left(\left.f_{t}\right|_{\Omega}\right)^{-1}\left(\phi_{t}(G)\right)$ we have $Q \circ \phi_{t}(z)=Q \circ f_{t}(z)$, which, by injectivity of $Q$ on the set $\phi_{t}(G)\left(\supset \phi_{t}\left(G_{0}\right), f_{t}\left(G_{0}\right)\right)$, leads to the conclusion that $\left.f_{t}\right|_{G_{0}}=\left.\phi_{t}\right|_{G_{0}}$, and consequently $\left.\mathcal{P}\right|_{G_{0}}=\left.\phi\right|_{G_{0}}$.

$\left(3^{\prime} .7\right)$ Theorem. Let $M \subset Z$ be a $C^{k}$-submanifold, $k \in\{2, \ldots, \infty, \omega\}$. Fix $z_{0} \in \operatorname{dom} \mathcal{P}$ and consider an inverse chart $f: \mathbb{R}^{n} \rightarrow M$ for which $f\left(x_{0}\right)=\mathcal{P}\left(z_{0}\right)$. Then the following conditions are equivalent:

(i) $z_{0} \in \Omega$;

(ii) $M$ is closed near $z_{0}$ (see (2.1)) and the matrix (3.12) is nonsingular.

Moreover, if one of these conditions is satisfied, then $\mathcal{P}$ is of class $C^{k-1}$ in a neighbourhood of $z_{0}$ and $\operatorname{im} d_{z_{0}} \mathcal{P}=T_{\mathcal{P}\left(z_{0}\right)} M$, $\operatorname{ker} d_{z_{0}} \mathcal{P}=T_{\mathcal{P}\left(z_{0}\right)}^{\perp} M$.

Proof. (i) $\Rightarrow$ (ii). According to Proposition (2.6), $M$ is closed near $z_{0}$. Suppose, contrary to our claim, that $\operatorname{det} A_{f}\left(z_{0}\right)=0$. This enables us to choose $\xi \in \mathbb{R}^{n}$ such that $|\xi|=1$ and

$$
\forall i: \quad \sum_{j}\left(\frac{\partial f}{\partial x_{i}}\left(x_{0}\right) \mid \frac{\partial f}{\partial x_{j}}\left(x_{0}\right)\right) \xi_{j}=\sum_{j}\left(\mathcal{P}\left(z_{0}\right)-z_{0} \mid \frac{\partial^{2} f}{\partial x_{i} \partial x_{j}}\left(x_{0}\right)\right) \xi_{j} .
$$

By the Taylor formula,

$$
\frac{f\left(x_{0}+t \xi\right)-f\left(x_{0}\right)-d_{x_{0}} f(t \xi)}{t^{2}}-\frac{1}{2 t^{2}} \underline{d}_{x_{0}}^{2} f(t \xi) \underset{t \rightarrow 0}{\longrightarrow} 0,
$$

where $\underline{d}_{x_{0}}^{2} f(t \xi):=d_{x_{0}}^{2} f(t \xi, t \xi)$. Computations using Remark (3.1) lead to the conclusion that

$$
\frac{\left(z_{0}-\mathcal{P}\left(z_{0}\right) \mid f\left(x_{0}+t \xi\right)-f\left(x_{0}\right)\right)}{t^{2}}-\frac{1}{2}\left|d_{x_{0}} f(\xi)\right|^{2} \underset{t \rightarrow 0}{\longrightarrow} 0 .
$$

Corollary (3.9) gives $\varepsilon>0$ such that $\mathcal{P}\left(\widetilde{z}_{0}\right)=\mathcal{P}\left(z_{0}\right)$ for $\widetilde{z}_{0}:=z_{0}+\varepsilon\left(z_{0}-\right.$ $\left.\mathcal{P}\left(z_{0}\right)\right)$. So for all $x \in \operatorname{dom} f$ we obtain

$$
\begin{aligned}
\left|\widetilde{z}_{0}-\mathcal{P}\left(z_{0}\right)\right|^{2} \leq & \left|\widetilde{z}_{0}-f(x)\right|^{2} \\
= & \left|\widetilde{z}_{0}-\mathcal{P}\left(z_{0}\right)\right|^{2}+2(1+\varepsilon)\left(z_{0}-\mathcal{P}\left(z_{0}\right) \mid f\left(x_{0}\right)-f(x)\right) \\
& +\left|f\left(x_{0}\right)-f(x)\right|^{2},
\end{aligned}
$$


which for $x=x_{0}+t \xi$ implies

$$
2(1+\varepsilon) \frac{\left(z_{0}-\mathcal{P}\left(z_{0}\right) \mid f\left(x_{0}+t \xi\right)-f\left(x_{0}\right)\right)}{t^{2}} \leq\left|\frac{f\left(x_{0}+t \xi\right)-f\left(x_{0}\right)}{t}\right|^{2}
$$

and further, as $t$ tends to zero,

$$
\varepsilon\left|\partial^{\xi} f\left(x_{0}\right)\right| \leq 0
$$

$\left(\partial^{\xi} f\left(x_{0}\right)\right.$ is the Gateaux derivative of $\left.f\right)$. This means that $d_{x_{0}} f$ is not a monomorphism, which is impossible.

(ii) $\Rightarrow\left(\right.$ i). Consider the $C^{k}$-mapping $B: Z \times \operatorname{dom} f \rightarrow \mathbb{R}^{n}$,

$$
B(z, x):=\left(\left(f(x)-z \mid \frac{\partial f}{\partial x_{1}}(x)\right), \ldots,\left(f(x)-z \mid \frac{\partial f}{\partial x_{n}}(x)\right)\right) .
$$

Notice that for $\left(z_{0}, x_{0}\right)(\in \operatorname{dom} B)$ the differential $d_{x_{0}} B\left(z_{0}, \cdot\right)$ is an automorphism of $\mathbb{R}^{n}$ (for $A_{f}\left(z_{0}\right)$ is its matrix in the canonical basis of $\mathbb{R}^{n}$ ). Since $B\left(z_{0}, x_{0}\right)=0$, there exists a $C^{k-1}$-map $\psi: Z \leftrightarrow \mathbb{R}^{n}$ such that $\psi\left(z_{0}\right)=x_{0}$ and $\psi \subset\{(z, x): B(z, x)=0\}$. Its differential $d_{z_{0}} \psi$ is an epimorphism. Therefore, the mapping $\phi:=f \circ \psi$, of class $C^{k-1}$, satisfies im $d_{z_{0}} \phi=T_{z_{0}} M$. Obviously, for all $i \in\{1, \ldots, n\}, \phi(z)-z \perp \frac{\partial f}{\partial x_{i}}(\phi(z))$ (see (3.1)), so $\phi$ satisfies the assumptions of Corollary (3.10) (see (3.6)). Thus $z_{0} \in \Omega$ (and $\phi=\mathcal{P}$ in a neighbourhood of $z_{0}$ ), which is our claim.

Now assume that either (i) or (ii) is satisfied. Repeating the construction from the proof of (ii) $\Rightarrow$ (i) we conclude that $\mathcal{P}(=\phi)$ is of class $C^{k-1}$ in a neighbourhood of $z_{0}$. We also know that $\operatorname{im} d_{z_{0}} \mathcal{P}=T_{z_{0}} M$. In order to find ker $d_{z_{0}} \mathcal{P}$ we choose $\mathcal{O} \in \operatorname{top} T_{\mathcal{P}\left(z_{0}\right)}^{\perp}$ such that $0 \in \mathcal{O}$ and $\mathcal{P} \equiv \mathcal{P}\left(z_{0}\right)$ on $z_{0}+\mathcal{O}$ (see (3.9)). For $u \in T_{\mathcal{P}\left(z_{0}\right)}^{\perp}$ such that $t u \in \mathcal{O}$, we have

$$
0=\frac{\mathcal{P}\left(z_{0}+t u\right)-\mathcal{P}\left(z_{0}\right)}{t} \underset{t \rightarrow 0}{\longrightarrow} \partial^{u} \mathcal{P}\left(z_{0}\right) .
$$

Thus $T_{\mathcal{P}\left(z_{0}\right)}^{\perp} \subset \operatorname{ker} d_{z_{0}} \mathcal{P}$. Also $\operatorname{dim} T_{\mathcal{P}\left(z_{0}\right)}^{\perp}=\operatorname{dim} \operatorname{ker} d_{z_{0}} \mathcal{P}$, so these spaces are indeed equal.

Proof of Theorem (3.11). This follows directly from Theorem $\left(3^{\prime} \cdot 7\right)$.

Proof of Theorem (3.13). (a) Fix $\left(a, a^{\prime}\right) \in Z \times M$ such that $\left|a-a^{\prime}\right|=$ $\varrho(a, M)$ and an inverse chart $f: \mathbb{R}^{n} \oplus M$ of class $C^{2}$ for which $a^{\prime} \in \operatorname{im} f$. For any $\left.z \in] a, a^{\prime}\right]$ consider the matrix (3.12) and the polynomial

$$
w:\left[0,1\left[\ni t \mapsto \operatorname{det} A_{f}\left(a^{\prime}+t\left(a-a^{\prime}\right)\right) .\right.\right.
$$

Clearly, $w(0) \neq 0$; thus $\#\{w=0\}<\infty$. There is $\delta>0$ such that $w(t) \neq 0$ for all $t \in] 1-\delta, 1[$. Fix $t \in] 1-\delta, 1\left[\right.$. Since $M$ is closed near $z_{t}:=a^{\prime}+t\left(a-a^{\prime}\right)$ (see $(2.7)), z_{t} \in \Omega$ (see (3.11)). We conclude from Theorem (1.5) that $\left[z_{t}, a^{\prime}[\subset \Omega\right.$ for all $t \in] 1-\delta, 1[$. Consequently, $\left.] a, a^{\prime}\right] \subset \Omega$. 
(b) Let $a \in \operatorname{dom} \mathcal{P}$ and $a^{\prime} \in M$.

(i) $\Rightarrow$ (ii). This results from (a) and Remark (3.1).

(ii) $\Rightarrow($ i). Assume that $\left.] a, a^{\prime}\right] \subset \Omega$ and $a-a^{\prime} \perp T_{a^{\prime}} M$. The set

$$
I:=\left\{t \in \left[0,1\left[: \mathcal{P}\left(a^{\prime}+t\left(a-a^{\prime}\right)\right)=a^{\prime}\right\}\right.\right.
$$

is non-empty $(0 \in I)$ and closed in $[0,1[$. It is also open in $[0,1[$, because for fixed $t \in I$ and $x:=a^{\prime}+t\left(a^{\prime}-a\right)(\in \Omega)$ one can choose $\delta>0$ such that $\mathcal{P}\left(a^{\prime}+(t+\delta)\left(a-a^{\prime}\right)\right)=\mathcal{P}\left(\delta\left(a-a^{\prime}\right)+x\right)=a^{\prime}$ (see (3.9)), which means that $\left[0, \min \{1, t+\delta\}\left[\subset I\right.\right.$. Hence $I=\left[0,1\left[\right.\right.$, i.e. $\mathcal{P}(z)=a^{\prime}$ for any $\left.\left.z \in\right] a, a^{\prime}\right]$. From this we deduce that $\varrho(a, M)=\left|a-a^{\prime}\right|$ and $\mathcal{P}(a)=a^{\prime}$.

Proof of Corollary (3.14). Fix $z \in \overline{\operatorname{dom} \mathcal{P}}$ and a sequence $\left(z_{\nu}\right) \in$ $(\operatorname{dom} \mathcal{P})^{\mathbb{N}}$ convergent to $z$. By Theorem $\left.\left.(3.13),\right] z_{\nu}, \mathcal{P}\left(z_{\nu}\right)\right] \subset \Omega$ for any $\nu \in \mathbb{N}$. Define

$$
x_{\nu}:=z_{\nu}+\frac{1}{\nu}\left(\mathcal{P}\left(z_{\nu}\right)-z_{\nu}\right) \in \Omega, \quad \nu=1,2, \ldots
$$

This sequence is convergent to $z$, since $\left(\left|z_{\nu}-\mathcal{P}\left(z_{\nu}\right)\right|\right)_{\nu=1}^{\infty}$ is bounded. Hence $\overline{\operatorname{dom} \mathcal{P}} \subset \bar{\Omega}(\subset \overline{\operatorname{dom} \mathcal{P}})$.

\section{$4^{\prime}$. Proofs}

Proof of Theorem (4.1). We only need to show (i) (see ( $\left.3^{\prime} .7\right)$ ). It follows from the equalities $\mathcal{P} \circ \mathcal{P}=\mathcal{P}$ and $d_{z} \mathcal{P} \circ d_{z} \mathcal{P}=d_{z} \mathcal{P}(\forall z \in M)$.

$\left(4^{\prime} .1\right)$ Lemma. Let $M \subset Z$ be a $C^{2}$-submanifold. Fix $z \in \Omega$ and an inverse chart $f: \mathbb{R}^{n} \leftrightarrow M$ of class $C^{2}$ such that $f(x)=\mathcal{P}(z)$. Let $\widetilde{A}_{f}(z)$ stand for the endomorphism of $\mathbb{R}^{n}$ given by the matrix (3.12) in the canonical basis. Then

$\forall \zeta \in Z: \quad d_{z} \mathcal{P}(\zeta)=\left(d_{x} f \circ \widetilde{A}_{f}(z)^{-1}\right)\left(\left(\zeta \mid \frac{\partial f}{\partial x_{1}}(x)\right), \ldots,\left(\zeta \mid \frac{\partial f}{\partial x_{n}}(x)\right)\right)$.

Proof. This follows from the proof of Theorem $\left(3^{\prime} .7\right)((\mathrm{ii}) \Rightarrow(\mathrm{i}))$.

The following two definitions are useful in formulating and proving the next theorems:

Let $X, Y$ be finite-dimensional real linear spaces. Fix $z \in X$, a subspace $H$ in $X$ and a mapping $g: X \leftrightarrow Y$ of a neighbourhood of $z$.

We say that $g$ is differentiable at $z$ with respect to $H$ iff $g \circ \tau_{z} \circ \iota_{H}$ is differentiable at zero (where $\iota_{H}: H \ni h \mapsto h \in X$, while $\tau_{z}$ denotes translation by $z)$. We write

$$
{ }^{H} d_{z} g:=d_{0}\left(g \circ \tau_{z} \circ \iota_{H}\right) .
$$

Next, we say that a sequence of linear operators $\alpha_{\nu}: X \oplus Y$ with non-zero domains $(\nu=1,2, \ldots)$ is convergent to a non-zero linear operator 
$\alpha: X \nrightarrow Y$ iff $\alpha_{\nu} \rightarrow \alpha$ as $\nu \rightarrow \infty$ in $X \times Y$ with respect to the Hausdorff metric.

We will use the following properties of this kind of convergence:

$\left(4^{\prime} .3\right)$ Let $l \in L(X, Y)$ and $\left(l_{\nu}\right) \in L(X, Y)^{\mathbb{N}}$. Then $l_{\nu} \rightarrow l$ in the Banach space $L(X, Y)$ iff $l_{\nu} \rightarrow l$ in the Hausdorff metric in $X \times Y$.

$\left(4^{\prime} .4\right)$ The map

$$
\left\{\begin{aligned}
\left.(A, B): \begin{array}{l}
A, B \text { are non-zero linear subspaces } \\
\text { of } X \text { with } A \cap B=0
\end{array}\right\} \ni(A, B) \\
\mapsto A+B \in\{E \neq 0: E \text { is a linear subspace of } X\}
\end{aligned}\right.
$$

is continuous.

$\left(4^{\prime} .5\right)$ Let $L: Y \rightarrow W$ be a linear operator with $W$ a finite-dimensional space, and let $\alpha: X \oplus Y$ be a partial linear operator. Also, let $\alpha_{\nu}: X \leftrightarrow Y$ and $L_{\nu}: Y \rightarrow W$ be linear operators $(\forall \nu \in \mathbb{N})$. If $\alpha_{\nu} \rightarrow \alpha$ and $L_{\nu} \rightarrow L$ as $\nu \rightarrow \infty$, then $L_{\nu} \circ \alpha_{\nu} \rightarrow L \circ \alpha$.

$\left(4^{\prime} .6\right)$ For a linear subspace $A$ of $X$ let $\iota_{A}: A \rightarrow X$ denote the canonical inclusion. Then the map

$\{$ non-zero linear subspaces of $X\} \ni B$

$$
\mapsto \iota_{B} \in\left\{\text { non-zero linear subspaces of } X^{2}\right\}
$$

is continuous.

The proofs of the above facts are based on the following criterion:

$\left(4^{\prime} .7\right)$ A function $f: X \rightarrow\{\alpha: Y \mapsto W \mid\{(0,0)\} \neq \alpha$ is linear $\}$ is continuous at $x_{0} \in X$ iff

$\forall \varepsilon>0 \exists \delta>0 \forall x \in X:\left\{\left|x-x_{0}\right| \leq \delta, v_{x} \in S \cap \operatorname{dom} f(x), v \in S \cap \operatorname{dom} f\left(x_{0}\right)\right.$,

$$
\left.\left|v-v_{x}\right| \leq \delta\right\} \Rightarrow\left|f(x)\left(v_{x}\right)-f\left(x_{0}\right)(v)\right| \leq \varepsilon,
$$

where $S$ stands for the unit sphere in $Y$. (Clearly, the last condition is independent of the choice of norms in $X, Y, W$.)

The criterion $\left(4^{\prime} .7\right)$ is a consequence of the following facts:

$\left(4^{\prime} .8\right)$ Let $C \subset X$ be a cone in $X$, i.e. by definition, $] 0, \infty[\cdot C \subset C$. If $C$ is open, then the class

$$
\widetilde{C}:=\left\{E \in \mathcal{G}_{p}(X): E \backslash\{0\} \subset C\right\}
$$

is open in the Grassmann manifold $\mathcal{G}_{p}(X)$ of p-dimensional subspaces of $X$.

(4'.9) Let a family $\left\{C_{\nu}\right\}_{\nu=1}^{\infty}$ of closed cones be a base of cone neighbourhoods of a subspace $E \in \mathcal{G}_{p}(X)$, i.e. by definition:

- $\forall \nu: C_{\nu+1} \subset C_{\nu}$ 
- $\forall \nu: E \backslash\{0\} \subset \operatorname{int} C_{\nu}$;

- $\bigcap_{\nu=1}^{\infty} C_{\nu}=E$.

Then $\left\{\widetilde{\operatorname{int}}_{\nu}\right\}_{\nu=1}^{\infty}$ is a neighbourhood base of $E$ in the topological space $\mathcal{G}_{p}(X)$.

Let $\alpha_{0}:=f\left(x_{0}\right): Y \leftrightarrow W$ be a partial linear operator from the criterion $\left(4^{\prime} .7\right)$, while $Q: Y \rightarrow \operatorname{dom} \alpha_{0}$ the linear orthogonal projection in the sense of a fixed inner product in $Y$. Then the family

$$
\begin{aligned}
C_{\nu}:=\{( & (y, w) \in Y \times W: \\
& \left.|| Q(y)|y-| y|Q(y)|+|| Q(y)\left|w-\alpha_{0}(|y| Q(y))\right| \leq \frac{1}{\nu}|y||Q(y)|\right\} \\
& \cap\left\{(y, w):\left|w-\alpha_{0}(Q(y))\right| \leq \frac{1}{2}|y| \leq|Q(y)|\right\}
\end{aligned}
$$

$(\nu=1,2, \ldots)$ of closed cones is a base of cone neighbourhoods of the subspace $\alpha_{0} \subset Y \times W$. The proof of $\left(4^{\prime} .7\right)$ rests on this fact.

(4'.10) Lemma. Let $M \subset Z$ be a $C^{2}$-submanifold. Let $z \in \Omega$ and let $f: \mathbb{R}^{n} \rightarrow M$ be an inverse chart for which $f(x)=\mathcal{P}(z)$. Fix $\zeta \in Z$ and put $\psi:=f^{-1} \circ \mathcal{P}_{\Omega}$. Then $\partial^{\zeta} \psi$ is differentiable at $z$ with respect to $T_{\mathcal{P}(z)}^{\perp}$ and for $v \in T_{\mathcal{P}(z)}^{\perp}$,

$T_{\mathcal{P}(z)}^{\perp} d_{z}\left(\partial^{\zeta} \psi\right)(v)=\partial^{v}\left(\partial^{\zeta} \psi\right)(z)=\sum_{i, j}\left(v \mid \frac{\partial^{2} f}{\partial x_{i} \partial x_{j}}(x)\right) \partial^{\zeta} \psi_{j}(z) \cdot \widetilde{A}_{f}(z)^{-1}\left(e_{i}\right)$,

where $\psi_{j}$ denotes the $j$-th coordinate function of $\psi,\left\{e_{1}, \ldots, e_{n}\right\}$ is the canonical basis in $\mathbb{R}^{n}$ and $\widetilde{A}_{f}(z)$ is the endomorphism of $\mathbb{R}^{n}$ with matrix $A_{f}(z)$ (see $(3.12))$, i.e. $\left(\widetilde{A}_{f}(z)\right)(\xi)=\sum_{i, j} A_{f}(z)_{i j} \cdot \xi_{j} e_{i}$ for $\xi \in \mathbb{R}^{n}$.

Proof. By (3.6) we can apply Corollary (3.9) to find $\mathcal{O} \in \operatorname{top} T_{\mathcal{P}(z)}^{\perp}$ such that $0 \in \mathcal{O}$ and $\mathcal{P} \equiv \mathcal{P}(z)$ on $z+\mathcal{O}$. In view of Lemma $\left(4^{\prime} .1\right)$ for every $v \in \mathcal{O}$ we have $z+v \in \operatorname{dom} \psi$ and

$$
\partial^{\zeta} \psi(z+v)=\widetilde{A}_{f}(z+v)^{-1}\left(\left(\zeta \mid \frac{\partial f}{\partial x_{1}}(x)\right), \ldots,\left(\zeta \mid \frac{\partial f}{\partial x_{n}}(x)\right)\right) .
$$

Also

$$
\widetilde{A}_{f}(z+v)=\widetilde{A}_{f}(z)-\sum_{i, j}\left(v \mid \frac{\partial^{2} f}{\partial x_{i} \partial x_{j}}(x)\right) e_{j}^{*} \cdot e_{i},
$$

where $\left\{e_{1}^{*}, \ldots, e_{n}^{*}\right\}$ is the dual basis to $\left\{e_{1}, \ldots, e_{n}\right\}$. The mapping $\mu:$ Aut $\mathbb{R}^{n}$ $\ni E \mapsto E^{-1} \in$ Aut $\mathbb{R}^{n}$ is analytic (as the solution of the implicit equation $R(E, \mu(E))=0$, where $\left.R:\left(\text { Aut } \mathbb{R}^{n}\right)^{2} \ni(E, F) \mapsto E \circ F-\mathrm{id}_{\mathbb{R}^{n}}\right)$. For $L \in$ End $\mathbb{R}^{n}$ we have $d_{E} \mu(L)=-E^{-1} \circ L \circ E^{-1}$. Also each $\xi \in \mathbb{R}^{n}$ defines the analytic mapping $\xi^{* *}$ : End $\mathbb{R}^{n} \in L \mapsto L(\xi) \in \mathbb{R}^{n}$. In this notation, for

$$
\xi:=\left(\left(\zeta \mid \frac{\partial f}{\partial x_{1}}(x)\right), \ldots,\left(\zeta \mid \frac{\partial f}{\partial x_{n}}(x)\right)\right)
$$


the relation $\left(4^{\prime} .11\right)$ takes the form

$$
\begin{aligned}
\partial^{\zeta} \psi(z+v) & =\left(\xi^{* *} \circ \mu\right)\left(\widetilde{A}_{f}(z+v)\right) \\
& =\left(\xi^{* *} \circ \mu\right)\left(\widetilde{A}_{f}(z)-\sum_{i, j}\left(v \mid \frac{\partial^{2} f}{\partial x_{i} \partial x_{j}}(x)\right) e_{j}^{*} \cdot e_{i}\right) .
\end{aligned}
$$

So $\partial^{\zeta} \psi$ is differentiable at $z$ with respect to $T_{\mathcal{P}(z)}^{\perp}$.

In order to find the explicit form of $\partial^{v}\left(\partial^{\zeta} \psi\right)(z)$ for $v \in T_{\mathcal{P}(z)}^{\perp}$, put

$$
\gamma(t):=\widetilde{A}_{f}(z)-t \sum_{i, j}\left(v \mid \frac{\partial^{2} f}{\partial x_{i} \partial x_{j}}(x)\right) e_{j}^{*} \cdot e_{i} \quad \text { for } t \in \mathbb{R} .
$$

Then

$$
\begin{aligned}
\partial^{v}\left(\partial^{\zeta} \psi\right)(z) & =\left.\frac{d}{d t} \partial^{\zeta} \psi(z+t v)\right|_{t=0}=\left(d_{\gamma(0)}\left(\xi^{* *} \circ \mu\right)\right)\left(\gamma^{\prime}(0)\right) \\
& =-\xi^{* *}\left(\gamma(0)^{-1} \circ \gamma^{\prime}(0) \circ \gamma(0)^{-1}\right) \\
& =\widetilde{A}_{f}(z)^{-1}\left(\sum_{i, j}\left(v \mid \frac{\partial^{2} f}{\partial x_{i} \partial x_{j}}(x)\right) \partial^{\zeta} \psi_{j}(z) \cdot e_{i}\right),
\end{aligned}
$$

which is the desired conclusion.

$\left(4^{\prime} .12\right)$ Lemma (the curvilinear version of the theorem on the existence of the Fréchet differential). Let $M \subset Z$ be a $C^{2}$-submanifold and $a \in M$. Let $Y$ denote a finite-dimensional linear space, and $g: Z \leftrightarrow Y$ a mapping of a neighbourhood of a such that $g_{M}:=\left.g\right|_{M}$ is differentiable at a. Assume that in a neighbourhood of a the differentials

$$
L_{z}:={ }^{T_{\mathcal{P}}^{\perp}(z)} d_{z} g
$$

exist, and

Then $g$ is differentiable at $a$.

$$
L_{z} \underset{z \rightarrow a}{\longrightarrow} L_{a}
$$

Pr o of. We will reduce this problem to the classical theorem. Consider a Euclidean space $X$ of dimension $\operatorname{dim} Z$, its subspace $H$ of $\operatorname{dimension} \operatorname{dim} M$ and an inverse chart $f: H \oplus M$ of class $C^{2}$ for which $f(0)=a$. There exists a $C^{2}$-diffeomorphism $\Phi: X \oplus Z$ such that $\Phi(0)=a,\left.f\right|_{H \cap \operatorname{dom} \Phi} \subset \Phi$ and for all $x \in \operatorname{dom} \Phi$, the map $H^{\perp} \ni v \mapsto \Phi(x+v)-\Phi(x)$ is contained in the linear isometry of $H^{\perp}$ and $T_{f\left(x^{\prime}\right)}^{\perp}$ (where $x^{\prime}$ stands for the orthogonal projection of $x$ onto $H$ ). In view of Theorem (3.8) we can assume that im $\Phi \subset \operatorname{dom} \mathcal{P}$ and $\mathcal{P}(z)=w$ whenever $z \in \operatorname{im} \Phi, w \in M \cap \operatorname{im} \Phi$ and $z-w \perp T_{w} M$. We will show that $g \circ \Phi$ satisfies the assumptions of the classical theorem on the existence of the Fréchet differential, which will complete the proof. 
Obviously, the differential ${ }^{H} d_{0}(g \circ \Phi)$ exists. Write $\mathcal{O}:=(\operatorname{dom} \Phi) \cap$ $\Pi^{-1}(\operatorname{dom} f \cap \operatorname{dom} \Phi)(\in \operatorname{top} X)$, where $\Pi: X \rightarrow H$ is the linear orthogonal projection. Also, fix $x \in \mathcal{O}$ and put $x^{\prime}:=\Pi(x)$. Then $\Phi(x)-f\left(x^{\prime}\right)=$ $\Phi\left(x^{\prime}+\left(x-x^{\prime}\right)\right)-\Phi\left(x^{\prime}\right) \in T_{f\left(x^{\prime}\right)}^{\perp}$, therefore $\mathcal{P}(\Phi(x))=f\left(x^{\prime}\right)$ and $L_{\Phi(x)}=$ $d_{0}\left(g \circ \tau_{\Phi(x)} \circ \iota_{T_{f\left(x^{\prime}\right)}^{\perp}}\right)$. Consider $u \in H^{\perp}$ such that $x+u \in \operatorname{dom} \Phi$. Then

$$
(g \circ \Phi)(u+x)=\left(g \circ \tau_{\Phi(x)} \circ \iota_{T_{f\left(x^{\prime}\right)}^{\perp}}\right)(\Phi(x+u)-\Phi(x)) .
$$

The differential of $H^{\perp} \ni u \mapsto \Phi(x+u)-\Phi(x)$ at zero is an isometry of $H^{\perp}$ and $T_{f\left(x^{\prime}\right)}^{\perp}$, contains the function itself and is the differential of $\Phi$ at $x$ with respect to $H^{\perp}$. Thus in a neighbourhood of zero we have

$$
(g \circ \Phi) \circ \tau_{x} \circ \iota_{H^{\perp}}=\left(g \circ \tau_{\Phi(x)} \circ \iota_{T_{f\left(x^{\prime}\right)}^{\perp}}\right) \circ d_{x} \Phi \circ \iota_{H^{\perp}},
$$

from which it follows that ${ }^{H^{\perp}} d_{x}(g \circ \Phi)$ exists for all $x \in \mathcal{O}$ and is equal to $\iota_{\Phi(x)} \circ d_{x} \Phi \circ \iota_{H^{\perp}}$.

Knowing that ${ }^{H} d_{0}(g \circ \Phi)$ exists we are reduced to proving that

$$
H^{\perp} d_{x}(g \circ \Phi) \underset{x \rightarrow 0}{\longrightarrow}{ }^{\perp} d_{0}(g \circ \Phi)
$$

in the Banach space $L\left(H^{\perp}, Y\right)$ or, which is equivalent, in the Hausdorff metric (see $\left(4^{\prime} .3\right)$ ). We will use the criterion $\left(4^{\prime} .7\right)$. Fix $\varepsilon>0$. Since $L_{z} \rightarrow L_{a}$ as $z \rightarrow a$, there is $\delta>0$ such that

$\forall|z-a| \leq \delta, v_{z} \in S \cap T_{f\left(z^{\prime}\right)}^{\perp}, v \in S \cap T_{a}^{\perp},\left|v-v_{z}\right| \leq \delta: \quad\left|L_{z}\left(v_{z}\right)-L_{a}(a)\right| \leq \varepsilon$.

Since $0 \in \mathcal{O}$ and $\Phi$ and $\Phi^{\prime}$ (the derivative of $\Phi$ ) are continuous, there is $\vartheta>0$ such that if $|x|<\vartheta$, then $x \in \mathcal{O},|\Phi(x)-a| \leq \delta$ and $\left|d_{x} \Phi-d_{0} \Phi\right| \leq \delta$. Now fix $x \in X$ such that $|x| \leq \delta$ and define $v_{\Phi(x)}:={ }^{H^{\perp}} d_{x} \Phi(u), v:={ }^{H^{\perp}} d_{0} \Phi(u)$. The functions $d_{x} \Phi \circ \iota_{H \perp}$ and $d_{0} \Phi \circ \iota_{H \perp}$ are isometries, therefore $\left|v_{\Phi(x)}\right|=|v|=1$. Hence $\left|{ }^{H^{\perp}} d_{x}(g \circ \Phi)(u)-{ }^{H^{\perp}} d_{0}(g \circ \Phi)(u)\right| \leq \varepsilon$.

$\left(4^{\prime} .13\right)$ Theorem (global version of $\left.\left(4^{\prime} .12\right)\right)$. Consider a $C^{2}$-submanifold $M \subset Z$ and a mapping $g: Z \leftrightarrow Y$ of a subset $\operatorname{dom} g \in \operatorname{top} Z$ of the domain of $\mathcal{P}$ with values in a finite-dimensional linear space $Y$. Suppose that $g_{M}:=\left.g\right|_{M}$ is of class $C^{1}$ and ${ }^{T_{\mathcal{P}(z)}^{\perp}} d_{z} g$ exists for any $z \in \operatorname{dom} g$. Moreover, assume that the function

$$
\operatorname{dom} g \ni z \mapsto{ }^{T_{\mathcal{P}}^{\perp}(z)} d_{z} g
$$

is continuous in the Hausdorff metric at any point of $M \cap \operatorname{dom} g$. Then

(i) $g$ is differentiable at a for all $a \in M \cap \operatorname{dom} g$;

(ii) the function $M \cap \operatorname{dom} g \ni a \mapsto d_{a} g$ is continuous.

Pr o of. By Lemma $\left(4^{\prime} .12\right)$ it remains to prove (ii). The map $G:=g_{M} \circ$ $\mathcal{P}_{\Omega}\left(\mathcal{P}_{\Omega}:=\left.\mathcal{P}\right|_{\Omega}\right)$ is of class $C^{1}$ (see (4.1)) and $M \cap \operatorname{dom} G=M \cap \operatorname{dom} g$. 
For $z \in M \cap \operatorname{dom} g$ we denote by $\alpha_{z}: T_{z} \hookrightarrow Z$ the inclusion. Theorem (4.1) states that $d_{z} \mathcal{P}$ is the orthogonal projection onto $T_{z}$, so

$$
\forall z \in M \cap \operatorname{dom} g: \quad d_{z} G \circ \alpha_{z}=d_{z} g \circ \alpha_{z}\left(={ }^{T_{z}} d_{z} g\right) .
$$

The map $M \ni z \mapsto T_{z} M$ is of class $C^{1}$. On the other hand, $\mathcal{G}_{\operatorname{dim} M}(Z) \ni$ $U \mapsto \iota_{U} \in L(U, Z)$ is continuous (see $\left(4^{\prime} .6\right)$ ). This yields the continuity of

$$
M \cap \operatorname{dom} G \ni z \mapsto d_{z} G \circ \alpha_{z}={ }^{T_{z}} d_{z} g \in \mathcal{G}_{\operatorname{dim} Z}(Z \times Y)
$$

(see $\left.\left(4^{\prime} .5\right)\right)$. From this we conclude that

$$
d_{z} g={ }^{T_{z}} d_{z} g \oplus^{T_{z}^{\perp}} d_{z} g \underset{M \ni z \mapsto a}{\longrightarrow}{ }^{T_{a}} d_{a} g \oplus^{T_{a}^{\perp}} d_{a} g=d_{a} g
$$

$\left(\operatorname{see}\left(4^{\prime} .4\right)\right)$.

Proof of Theorem (4.2). It is sufficient to show that the assertion holds locally. Let $f: \mathbb{R}^{n} \oplus M$ be an inverse chart of class $C^{2}$ and, according to the notation of Lemma $\left(4^{\prime} .10\right)$, set $\psi:=f^{-1} \circ \mathcal{P}$. This is a $C^{1}$-mapping. The proof will be completed when we prove that for any $\zeta \in Z, \partial^{\zeta} \psi$ is differentiable and the function $\operatorname{im} f \ni x \mapsto d_{x}\left(\partial^{\zeta} \psi\right)$ is continuous.

Fix $\zeta \in Z$. Intending to make use of Theorem $\left(4^{\prime} .13\right)$ we have to show that $\left(\partial^{\zeta} \psi\right)_{M}$ is of class $C^{1}$ and that for $z \in \operatorname{dom} \psi$, the differentials $T_{\mathcal{P}(z)}^{\perp} d_{z}\left(\partial^{\zeta} \psi\right)$ exist and converge to ${ }^{T_{z_{0}}^{\perp}} d_{z_{0}}\left(\partial^{\zeta} \psi\right)$ as $z \rightarrow z_{0}(\in M)$. In the notation of $\left(4^{\prime} .11\right)$,

$$
\begin{aligned}
\partial^{\zeta} \psi(z) & \left(\sum_{i, j}\left(\frac{\partial f}{\partial x_{i}}(x) \mid \frac{\partial f}{\partial x_{j}}(x)\right) e_{j}^{*} \cdot e_{i}\right)\left(\left(\zeta \mid \frac{\partial f}{\partial x_{1}}(x)\right), \ldots,\left(\zeta \mid \frac{\partial f}{\partial x_{n}}(x)\right)\right)
\end{aligned}
$$

for $z=f(x)(x \in \operatorname{dom} f)$. The right-hand side is a $C^{1}$-function of $x$, thus $\left(\partial^{\zeta} \psi\right)_{M}$ is of class $C^{1}$. Now we only have to show that

$$
T_{\mathcal{P}(z)}^{\perp} d_{z}\left(\partial^{\zeta} \psi\right) \underset{z \rightarrow z_{0}}{\longrightarrow} T_{z_{0}}^{\perp} d_{z_{0}}\left(\partial^{\zeta} \psi\right)
$$

where $z_{0} \in \operatorname{im} f$. This follows from the criterion $\left(4^{\prime} .7\right)$ and from $T_{\mathcal{P}(z)} \rightarrow T_{z_{0}}$ and $T_{\mathcal{P}(z)}^{\perp} \rightarrow T_{z_{0}}^{\perp}$ as $z \rightarrow z_{0}($ see $(3.5))$.

Discussion of Example (4.3). Consider the $C^{2}$-embedding $f$ : $\mathbb{R} \rightarrow \mathbb{R}^{2}$ given by

$$
f(t):= \begin{cases}\left(t, \frac{1}{3} t^{3}\right), & t \geq 0 \\ (t, 0), & t<0\end{cases}
$$

and the submanifold $M:=f(\mathbb{R})$. Suppose, contrary to our claim, that

$\left(4^{\prime} .14\right) \mathcal{P}$ is twice differentiable in a neighbourhood of $(0,0)$.

Then there is $r>0$ such that $\mathcal{P}$ is twice differentiable in $U:=]-r, r\left[^{2}\right.$ and

$$
\forall z \in U \forall w \in U \cap M: \quad z-w \perp T_{w} M \Rightarrow \mathcal{P}(z)=w
$$


(see (3.8)). Thus, for $z=\left(z_{1}, z_{2}\right) \in U \cap\{(x, y): x \leq 0\}$ we have

$$
z_{1}=\left(f^{-1} \circ \mathcal{P}\right)(z) \text {. }
$$

Fix $\lambda \in] 0, r\left[\right.$ and define the $C^{2}$-curve

$$
g_{\lambda}: \mathbb{R} \ni t \mapsto f(t)+\left(-\lambda t^{2}, \lambda\right) .
$$

There is $\delta>0$ such that for all $t \in[0, \delta], t^{4}-2 \lambda t+1>0$ and $g_{\lambda}(t), f(t) \in U$ and $\mathcal{P}\left(g_{\lambda}(t)\right)=f(t)$. In view of $\left(4^{\prime} .14\right)$ the map

$$
\kappa(t):=\partial^{e_{1}}\left(f^{-1} \circ \mathcal{P}_{\Omega}\right)\left(g_{\lambda}(t)\right)
$$

is differentiable in a neighbourhood of zero. We have $\operatorname{det} A_{f}\left(g_{\lambda}(t)\right)=t^{4}-$ $2 \lambda t+1 \neq 0$ for $t \in[0, \delta]$ (see (3.12)), so by $\left(4^{\prime} .11\right), \kappa(t)=1 /\left(t^{4}-2 \lambda t+1\right)$ for any $t \in[0, \delta]$. Therefore $\kappa^{\prime}\left(0^{+}\right)=2 \lambda \neq 0$. On the other hand, $\left(4^{\prime} .15\right)$ yields $\partial^{e_{1}}\left(f^{-1} \circ \mathcal{P}_{\Omega}\right) \equiv 1$ in $U \cap\{(x, y): x \leq 0\}$, thus $\kappa^{\prime}\left(0^{-}\right)=0$, a contradiction.

Proof of Proposition (4.4). The mapping

$$
\varrho: \Omega \backslash M \ni x \mapsto|x-\mathcal{P}(x)| \in \mathbb{R}
$$

is of class $C^{1}$. For all $b \in Z \backslash\{0\}$ one obtains

$$
\begin{aligned}
\partial^{b} \varrho^{2}(x) & =2\left(x-\mathcal{P}(x) \mid b-\partial^{b} \mathcal{P}(x)\right) \\
& =2(x-\mathcal{P}(x) \mid b)-2\left(x-\mathcal{P}(x) \mid d_{x} \mathcal{P}(b)\right) \\
& =2(x-\mathcal{P}(x) \mid b),
\end{aligned}
$$

since $x-\mathcal{P}(x) \in T_{\mathcal{P}(x)}^{\perp}$ and $d_{x} \mathcal{P}(b) \in T_{\mathcal{P}(x)}$. On the other hand, $\partial^{b}\left(\lambda^{2}\right)=$ $2 \varrho \cdot \partial^{b} \varrho$, so $2 \varrho(x) \cdot \partial^{b} \varrho(x)=2(x-\mathcal{P}(x) \mid b)$, and finally,

$$
\partial^{b} \varrho(x)=\left(\frac{x-\mathcal{P}(x)}{|x-\mathcal{P}(x)|} \mid b\right) \text {. }
$$

\section{5 . Proofs}

$\left(5^{\prime} .1\right)$ Remark. For every submanifold $M \subset Z$ the following conditions are equivalent:

(i) $M$ is non-empty, convex and closed;

(ii) $M$ is an affine subspace of $Z$.

Proof. (ii) $\Rightarrow$ (i) is obvious.

(i) $\Rightarrow$ (ii). One can assume that $0 \in M$. Let $H:=\operatorname{Lin} M, n:=\operatorname{dim} H$ $(\geq \operatorname{dim} M),\left\{e_{1}, \ldots, e_{n}\right\} \subset M$ be a basis of $H$ and $\left\{e_{1}^{*}, \ldots, e_{n}^{*}\right\}$ the dual basis. The set

$$
\begin{aligned}
\left\{h \in H:\left(e_{1}^{*}+\ldots+e_{n}^{*}\right)(h)<1\right\} \cap \bigcap_{i=1}^{n}\left\{h \in H: e_{i}^{*}(h)>0\right\} & \\
& \subset \operatorname{conv}\left\{0, e_{1}, \ldots, e_{n}\right\}
\end{aligned}
$$


is non-empty, open in $H$ and contained in $M$. Therefore $\operatorname{dim} M=n$, and consequently $M \in$ top $H$. Finally, $H=M$.

Proof of Theorem (5.3). This follows (even for $M$ being a $C^{1}$ submanifold) immediately from $(5.2)$ and $\left(5^{\prime} .1\right)$. We will present a more elementary proof without the use of Theorem (5.2). The set $M$ is closed, because if $\left(x_{n}\right) \in M^{\mathbb{N}}$ converges to $z \in Z$, then $0=\left|x_{n}-\mathcal{P}\left(x_{n}\right)\right| \rightarrow|z-\mathcal{P}(z)|$ as $n \rightarrow \infty$. Hence $z=\mathcal{P}(z) \in M$. In view of Remark (5'.1) it suffices to prove the convexity of $M$. Fix $a \in Z$ and set

$$
J:=\left\{t \in \mathbb{R}_{+}: \mathcal{P}(\mathcal{P}(a)+t(a-\mathcal{P}(a)))=\mathcal{P}(a)\right\},
$$

where $\mathbb{R}_{+}:=\left[0, \infty\left[\right.\right.$. Notice that $J \in\left(\operatorname{cotop} \mathbb{R}_{+}\right) \backslash\{\emptyset\}$. Simultaneously $J \in$ top $\mathbb{R}_{+}$. In order to prove this, fix $t_{0} \in J$ and put $b:=\mathcal{P}(a)+t_{0}(a-\mathcal{P}(a))$. By Corollary (3.9) there exists $\mathcal{O} \in \operatorname{top} T_{\mathcal{P}(a)}^{\perp}$ such that $0 \in \mathcal{O}$ and $\mathcal{P} \equiv$ $\mathcal{P}(a)$ on $b+\mathcal{O}$. Moreover, there is $\delta>0$ for which $\delta(a-\mathcal{P}(a)) \in \mathcal{O}$. Thus $\mathcal{P}(a)=\mathcal{P}(b+\delta(a-\mathcal{P}(a)))$, which means that $\left[0, t_{0}+\delta\right] \subset J$. Consequently, $J=\mathbb{R}_{+}$.

Now we show that $M$ is contained in the half-space $\Pi(a):=\{z \in Z$ : $(z-\mathcal{P}(a) \mid a-\mathcal{P}(a)) \leq 0\}$. Fix $x \in M$ and $t \in J\left(=\mathbb{R}_{+}\right)$. We have

$$
|\mathcal{P}(a)+t(a-\mathcal{P}(a))-x| \geq|\mathcal{P}(a)+t(a-\mathcal{P}(a))-\mathcal{P}(a)|,
$$

which is equivalent to the inequality

$$
(x-\mathcal{P}(a) \mid a-\mathcal{P}(a)) \leq \frac{1}{2 t}|\mathcal{P}(a)-x|^{2}
$$

for all $t>0$, so indeed $x \in \Pi(a)(\forall a \in Z)$. Therefore $M \subset \bigcap_{a \in Z} \Pi(a)$. The inverse inclusion also holds, since for $z \in \bigcap_{a \in Z} \Pi(a)$ we have, in particular, $z \in \Pi(z)$. This implies $z=\mathcal{P}(z) \in M$. As a consequence, $M\left(=\bigcap_{a \in Z} \Pi(a)\right)$ is convex.

\section{$6^{\prime}$. Proofs}

$\left(6^{\prime} .1\right)$ Remark. Consider a concave function $f: \mathbb{R}^{k} \rightarrow \mathbb{R}$ (i.e. such that the set $\left\{(x, y) \in \mathbb{R}^{k} \times \mathbb{R}: f(x) \geq y\right\}$ is convex) and the orthogonal projection $\mathcal{P}: \mathbb{R}^{k} \times \mathbb{R} \Leftrightarrow f$. Then $\left\{(x, y) \in \mathbb{R}^{k} \times \mathbb{R}: f(x) \leq y\right\} \subset \operatorname{dom} \mathcal{P}$.

$\left(6^{\prime} .2\right)$ Remark. Consider a Euclidean space $Z$, a set $M \subset Z$, the orthogonal projection $\mathcal{P}: Z \leftrightarrow M$ and a subset $U \subset Z$. Assume that $I: Z \rightarrow Z$ is an isometry such that $I(M)=M$. Then $I(U \backslash \operatorname{dom} \mathcal{P})=I(U) \backslash \operatorname{dom} \mathcal{P}$.

Let us introduce the symbols and notions constantly used throughout this section. We view $Z=\mathbb{R}^{2}$ as the complex plane $\mathbb{C}$. For all $a \in \mathbb{R}$ for which the following definition makes sense, we denote by $L(a)$ the affine line $(a+f(a) i)+T_{a+f(a) i}^{\perp} M$, where $f: \mathbb{R} \leftrightarrow \mathbb{R}$ is the function under consideration and $M(=f)$ is its graph. 
Discussion of Example (6.1). For $a \in \mathbb{R} \backslash\{0\}$ the normal line $L(a)=\left\{x+i y: y-a^{2}=\frac{-1}{2 a}(x-a)\right\}$ intersects $\mathbb{R} i$ at $\left(a^{2}+1 / 2\right) i$. Therefore $[0, i / 2] \subset \operatorname{dom} \mathcal{P}$ and $\left.\mathcal{P}\right|_{[0, i / 2]} \equiv 0$. Also, for $\left.z \in\right] 1 / 2, \infty[\cdot i$ there exists a unique $a>0$ such that $z \in L(a)$. Moreover, $\{b \in \mathbb{R}: z \in L(b)\}=\{-a, 0, a\}$. But since $\left|a+a^{2} i-z\right|=\left|-a+a^{2} i-z\right|<|z|$, we have $z \notin \operatorname{dom} \mathcal{P}$. Hence

$$
\mathbb{R}_{+} i \cap \operatorname{dom} \mathcal{P}=[0, i / 2] \text {. }
$$

By Remark (6'.1), $\left\{x+i y: y \leq x^{2}\right\} \subset \operatorname{dom} \mathcal{P}$. We are left with considering points $z_{0}=x_{0}+i y_{0}$ for which $x_{0} \neq 0$ and $y_{0}>x_{0}^{2}$. For such $z_{0}$ there exists $a+a^{2} i \neq 0$ realizing the distance of $z_{0}$ from $f$. Thus $\left.z_{0} \in\right] a+a^{2} i,\left(a^{2}+1\right) i[\subset$ $\operatorname{dom} \mathcal{P}$ and $\mathcal{P}\left(z_{0}\right)=a+a^{2} i$ (see (1.5)). Finally, $\operatorname{dom} \mathcal{P}=\mathbb{C} \backslash(] 1 / 2, \infty[\cdot i)$.

Discussion of Example (6.2). The results obtained in the discussion of Example (6.1) imply

$$
\{z \in \mathbb{C}: \operatorname{Re} z \geq 0\} \subset \operatorname{dom} \mathcal{P} .
$$

Next, for $z=x+i y$ such that $x<0$ define

$h=h_{z}:\left[0, \infty\left[\ni a \mapsto\left|x+i y-\left(a+a^{2} i\right)\right|^{2}=a^{4}+(1-2 y) a^{2}-2 a x+x^{2}+y^{2}\right.\right.$,

which is either increasing or reaches one minimum. Therefore

$$
x+i y \notin \operatorname{dom} \mathcal{P} \Leftrightarrow \exists a_{0}>0: h^{\prime}\left(a_{0}\right)=0 \text { and } h(0)=h\left(a_{0}\right) .
$$

The system of equations on the right-hand side of the above equivalence has a solution $a_{0}>0$ iff $y=\frac{1}{2}\left(3(-x)^{2 / 3}+1\right)$. Then $a_{0}=(-x)^{1 / 3}$. So the graph of the function

$$
G:]-\infty, 0\left[\ni x \mapsto \frac{1}{2}\left(3(-x)^{2 / 3}+1\right) \in \mathbb{R}\right.
$$

coincides with $\mathbb{R}^{2} \backslash \operatorname{dom} \mathcal{P}$.

Discussion of Example (6.3). When we delete zero from the $M$ of Example (6.2), all the points that were previously projected onto zero disappear from $\operatorname{dom} \mathcal{P}$. These are exactly the points $z \in\{w: \operatorname{Re} w \leq 0\}$ for which, in the notation of Discussion of $(6.2)$, either $z \in]-\infty, 1 / 2[\cdot i$, or $h^{\prime}\left(a_{0}\right)=0$ and $h(0)=h\left(a_{0}\right)$ for some $a_{0}>0$. Finally,

$$
\operatorname{dom} \mathcal{P}=\mathbb{C} \backslash(\{i / 2\} \cup\{x+i y: x \leq 0, y<G(x)\}) \quad\left(\text { see }\left(6^{\prime} .3\right)\right) .
$$

Discussion of Example (6.4). First consider points of $\mathbb{R} i$. If $a+$ $i(\cos a)$ realizes such a point's distance from $f=\cos$, then $|a|<\pi$. The normal at $a+i(\cos a)$,

$$
L(a)=\left\{x+i y: y-\cos a=\frac{x-a}{\sin a}\right\} \quad(\text { for } 0<|a|<\pi),
$$

intersects $\mathbb{R} i$ at $y(a):=(\cos a-a / \sin a) i$. Since the function

$$
y:] 0, \pi[\ni a \mapsto y(a) \in]-\infty, 0[\cdot i
$$


is continuous and bijective, for any $z \in[0, \infty[\cdot i$ the point $a=0$ is the unique point $a$ in $]-\pi, \pi$ [ for which $z$ lies on $L(a)$. Therefore $[0, \infty[\cdot i \subset \operatorname{dom} \mathcal{P}$.

Next, for fixed $y_{0}<0$ there exists a unique $\left.a \in\right] 0, \pi\left[\right.$ such that $y_{0} i \in$ $L(a)$. Simultaneously, $\{b \in]-\pi, \pi\left[: y_{0} i \in L(b)\right\}=\{-a, 0, a\}$. If we had $\left|y_{0} i-(a+i \cos a)\right|>\left|y_{0} i-i\right|$, then $y_{0} i \in \operatorname{dom} \mathcal{P}$ and $\mathcal{P}\left(y_{0} i\right)=i$, hence $\left.0 \in] y_{0} i, i\right] \subset \Omega$ (see (3.13)) and, by Theorem (3.11), $0 \neq \operatorname{det} A_{F}(0)$, where $F: \mathbb{R} \ni a \mapsto(a, \cos a) \in \mathbb{R}^{2}$. But the matrix $A_{F}(0)$ is singular. Therefore, the distance of $y_{0} i$ from $f$ is realized by two points: $a+i \cos a,-a+i \cos a$. This means that $\mathbb{R} i \cap \operatorname{dom} \mathcal{P}=[0, \infty[\cdot i$.

Now, consider $z_{0}=x_{0}+i y_{0}$ such that $\left.x_{0} \in\right] 0, \pi\left[\right.$ and $y_{0}<\cos x_{0}$. As mentioned at the beginning, there exists $a \in] 0, \pi[$ such that $a+i \cos a$ realizes the distance of $z_{0}$ from $f$. But $\left.z_{0} \in\right] y(a), a+i \cos a[\subset \operatorname{dom} \mathcal{P}$. Therefore, for

$$
U:=\mathbb{R} i \cup\{x+i y: x \in] 0, \pi[, y \leq \cos x\}
$$

we have $U \backslash \operatorname{dom} \mathcal{P}=]-\infty, 0[\cdot i$. Put $M:=f$. Consider the family $\mathcal{J}:=$ $\left\{\tau_{2 k \pi} \circ I\right\}_{k \in \mathbb{Z}}$ of isometries of the plane $\mathbb{C}$, where $\tau_{2 k \pi}$ is translation by $2 k \pi$, while $I$ is either $\operatorname{id}_{\mathbb{C}}, S_{\pi+\mathbb{R} i}, R_{\pi / 2}$, or $S_{\pi+\mathbb{R} i} \circ R_{\pi / 2}$ (where $S_{b}$ is the symmetry about a straight line $b$ and $R_{B}$ the symmetry about a point $B$ ). Then

$$
\bigcup_{J \in \mathcal{J}} J(U)=\mathbb{C} \quad \text { and } \quad \forall J \in \mathcal{J}: J(M)=M
$$

Applying Remark $\left(6^{\prime} .2\right)$ to our $U$ we obtain

$$
\mathbb{C} \backslash \operatorname{dom} \mathcal{P}=\left(\bigcup_{J \in \mathcal{J}} J(U)\right) \backslash \operatorname{dom} \mathcal{P}=\bigcup_{k \in \mathbb{Z}}(k \pi+] 0, \infty\left[\cdot(-1)^{k+1} i\right) .
$$

Discussion of Example (6.5). Remark (6'.1) yields $\{x+i y: y \leq$ $\left.e^{x}\right\} \subset \operatorname{dom} \mathcal{P}$. Fix $z=x+i y \in \mathbb{C}$ and consider the function

$$
h=h_{z}: \mathbb{R} \ni a \mapsto\left|z-\left(a+i e^{a}\right)\right|^{2}=(x-a)^{2}+\left(y-e^{a}\right)^{2} .
$$

For $|y| \leq 2 \sqrt{2}, h$ reaches one minimum, so $\{w \in \mathbb{C}: \operatorname{Im} w \leq 2 \sqrt{2}\} \subset \operatorname{dom} \mathcal{P}$. If $y=\operatorname{Im} z>2 \sqrt{2}$, then $h^{\prime \prime}(a)=0$ has two solutions $a_{1}<a_{2}$. A sufficient condition for $z \notin \operatorname{dom} \mathcal{P}$ is:

$$
h^{\prime}\left(a_{1}\right)>0 \text { and } h^{\prime}\left(a_{2}\right)<0 .
$$

This is equivalent to

$$
-\ln \sqrt{2 e}-u_{2}(t)<x<-\ln \sqrt{2 e}-u_{1}(t),
$$

where $t:=y / 2 \sqrt{2}(>1)$ and

$$
\begin{aligned}
& u_{1}(t):=t\left(t-\sqrt{t^{2}-1}\right)+\ln \left(t+\sqrt{t^{2}-1}\right), \\
& u_{2}(t):=t\left(t+\sqrt{t^{2}-1}\right)-\ln \left(t+\sqrt{t^{2}-1}\right) .
\end{aligned}
$$

Moreover, $\lim _{t \rightarrow \infty} u_{1}(t)=\lim _{t \rightarrow \infty} u_{2}(t)=\infty$ and the graphs of $u_{1}$ and $u_{2}$ 
are tangent at the point corresponding to $t=1$, i.e. at $-1-\ln \sqrt{2 e}+2 \sqrt{2} i$. The condition $\left(6^{\prime} .4\right)$ is satisfied by points of the set

$$
\left\{x+i y: y>2 \sqrt{2} \text { and }-u_{2}(y / 2 \sqrt{2})<x<-u_{1}(y / 2 \sqrt{2})\right\}
$$

which contains $\mathbb{C} \backslash$ dom $\mathcal{P}$.

\section{References}

[1] E. Asplund, Čebyšev sets in Hilbert space, Trans. Amer. Math. Soc. 144 (1969), 235-240.

[2] L. N. H. Bunt, Contributions to the theory of convex point sets, Ph.D. Thesis, Groningen, 1934 (in Dutch).

[3] E. Dudek, Orthogonal projection onto a subset of a Euclidean space, Master's thesis, Kraków, 1989 (in Polish).

[4] N. V. Efimov and S. B. Stechkin, Support properties of sets in Banach spaces and Chebyshev sets, Dokl. Akad. Nauk SSSR 127 (1959), 254-257 (in Russian).

[5] H. Federer, Curvature measures, Trans. Amer. Math. Soc. 93 (1959), 418-491.

[6] D. Gilbarg and N. Trudinger, Elliptic Partial Differential Equations of Second Order, Springer, Berlin, 1977.

[7] M. W. Hirsch, Differential Topology, Springer, New York, 1976.

[8] E. Hopf, On non-linear partial differential equations, in: Lecture Series of the Symposium on Partial Diff. Equations, Berkeley, 1955, The Univ. of Kansas, 1957, 1-29.

[9] G. Jasiński, A characterization of the differentiable retractions, Univ. Iagell. Acta Math. 26 (1987), 99-103.

[10] V. L. Klee, Convexity of Chebyshev sets, Math. Ann. 142 (1961), 292-304.

[11] - Remarks on nearest points in normed linear spaces, in: Proc. Colloquium on Convexity (Copenhagen, 1965), Kobenhavns Univ. Mat. Inst., Copenhagen, 1967, $168-176$.

[12] S. G. Krantz and H. R. Parks, Distance to $C^{k}$ hypersurfaces, J. Differential Equations 40 (1981), 116-120.

[13] J. L. Lions, Quelques méthodes de résolution des problèmes aux limites non linéaires, Dunod and Gauthier-Villars, Paris, 1969.

[14] T. Motzkin, Sur quelques propriétés caractéristiques des ensembles convexes, Atti R. Accad. Lincei Rend. (6) 21 (1935), 562-567.

[15] W. Rudin, Real and Complex Analysis, McGraw-Hill, New York, 1974.

[16] J. Serrin, The problem of Dirichlet for quasilinear elliptic differential equations with many independent variables, Philos. Trans. Roy. Soc. London Ser. A 264 (1969), 413-496.

Ewa Dudek

INSTITUTE OF MATHEMATICS

PEDAGOGICAL UNIVERSITY

PODCHORA̧ŻYCH 2

30-084 KRAKÓW, POLAND
Konstanty Holly

INSTITUTE OF MATHEMATICS JAGIELLONIAN UNIVERSITY REYMONTA 4 30-059 KRAKÓW, POLAND 